Provided for non-commercial research and education use. Not for reproduction, distribution or commercial use.

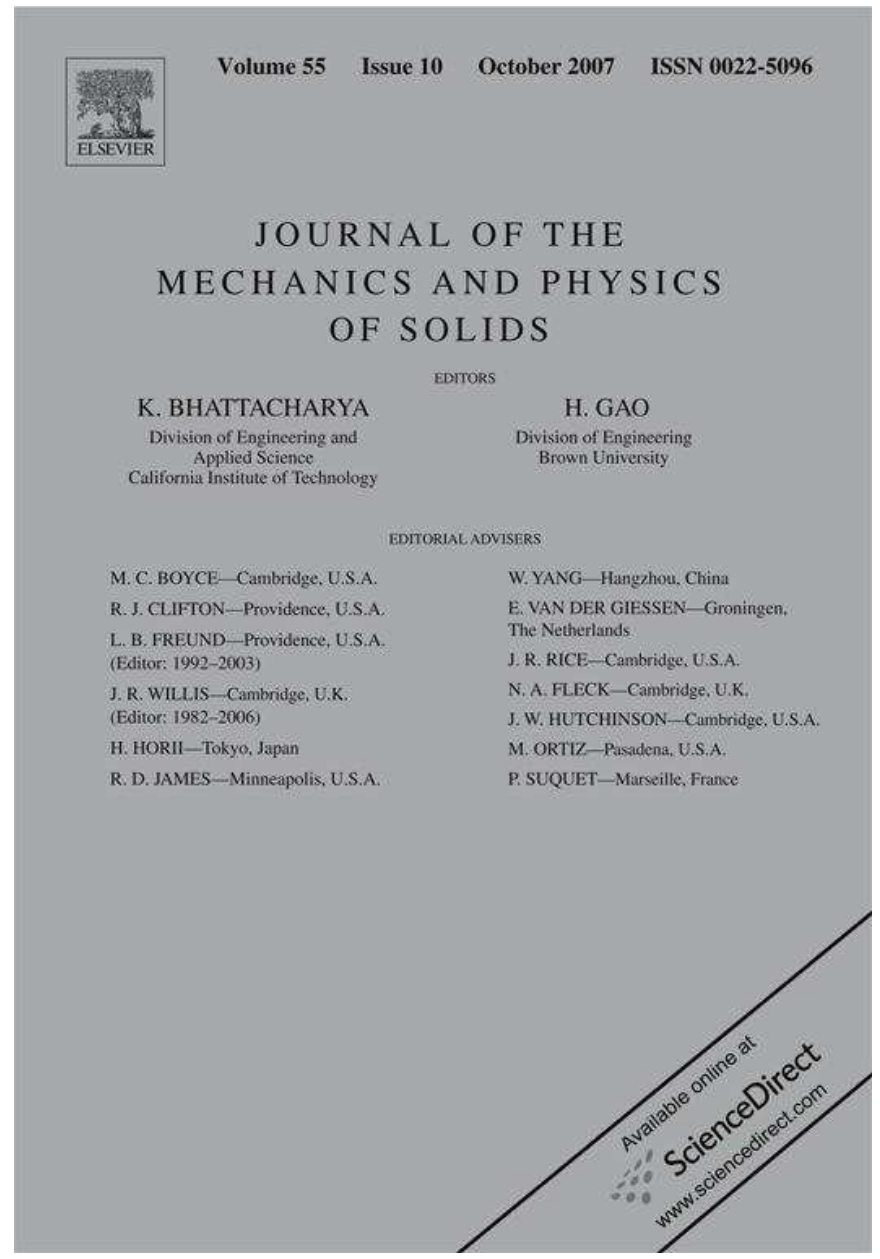

This article was published in an Elsevier journal. The attached copy

is furnished to the author for non-commercial research and education use, including for instruction at the author's institution, sharing with colleagues and providing to institution administration.

Other uses, including reproduction and distribution, or selling or licensing copies, or posting to personal, institutional or third party websites are prohibited.

In most cases authors are permitted to post their version of the article (e.g. in Word or Tex form) to their personal website or institutional repository. Authors requiring further information regarding Elsevier's archiving and manuscript policies are encouraged to visit:

http://www.elsevier.com/copyright 


\title{
Sliding modes of two interacting frictional interfaces
}

\author{
Thibaut Putelat ${ }^{\mathrm{a}, \mathrm{b}, *}$, Jonathan H.P. Dawes ${ }^{\mathrm{b}}$, John R. Willis ${ }^{\mathrm{b}}$ \\ ${ }^{a}$ Institute of Theoretical Geophysics, University of Cambridge, Centre for Mathematical Sciences, \\ Wilberforce Road, Cambridge CB3 OWA, UK \\ ${ }^{\mathrm{b}}$ Department of Applied Mathematics \& Theoretical Physics, University of Cambridge, \\ Centre for Mathematical Sciences, Wilberforce Road, Cambridge CB3 OWA, UK
}

Received 10 October 2006; received in revised form 2 March 2007; accepted 16 March 2007

\begin{abstract}
In the context of rate-and-state friction, we report an extensive analysis of stability of the quasi-static frictional sliding of two parallel interfaces dividing a linear elastic solid sheared at a constant rate. One possibility for the frictional sliding is that the interfaces slip at equal rates, a steady state described as symmetric. However a steady-state friction law that is non-monotonic allows the competing possibility of an asymmetric steady state in which the interfaces slide at different rates. A rate-and-state law that delivers such behaviour and agrees with the experimental results of Heslot et al. [1994. Creep, stick-slip, and dryfriction dynamics: experiments and a heuristic model. Phys. Rev. E 49, 4973-4988] is proposed. Analytical results combined with numerical investigations performed with the continuation package AuTo and direct time integration are used to compile the complete picture of the many bifurcations that exist between the diverse steady and oscillatory sliding modes. In addition to the control parameters corresponding to the driving velocity and the stiffness of the medium, we find that the geometrical details of the steady-state friction law determine the occurrence and nature of bifurcations. Pitchfork bifurcations from the symmetric to asymmetric steady states coincide with the extrema of the friction law; Hopf bifurcations occur in the velocity weakening regime of the friction law. Torus and period-doubling bifurcations of periodic orbits also occur, and lead to complicated dynamics. We also present results of numerical computations that illustrate the complex and versatile dynamics of the two-interface system. We anticipate that the dynamics found in our model should be verifiable by experiments.
\end{abstract}

(C) 2007 Elsevier Ltd. All rights reserved.

Keywords: Rate-and-state friction; Non-monotonicity; Stick-slip oscillation; Nonlinear bifurcation analysis; Continuation methods

\footnotetext{
*Corresponding author.

E-mail address: T.Putelat@damtp.cam.ac.uk (T. Putelat).
} 


\section{Introduction}

Motivated by the question of how active fault planes are selected in a network of faults, we study the stability of the quasi-static sliding of two parallel frictional interfaces which divide an elastic body sheared at a constant velocity applied at one edge of the medium. Our first concern is to determine under which conditions different steady states can exist and be stable in the framework of rate-and-state friction originally developed in geophysical studies (Ruina, 1983; Marone, 1998; Scholz, 1998). In this context, when the sliding is steady with a homogeneous shear stress, and if the steady-state friction coefficient is a non-monotonic function of the slip velocity, we expect that the interfaces are able to slide with two different slip rates, producing slow and fast interfaces.

Such a non-monotonic behaviour of friction has been known for a long time (e.g. Bowden and Tabor, 1954; Rabinowicz, 1995). In particular, concerning rock friction, several transitions between velocity weakening and strengthening have been experimentally observed (Shimamoto, 1986; Kilgore et al., 1993; Tsutsumi and Shimamoto, 1997). These transitions occur because the friction processes involved during the sliding differ according to the slip rate at which the interface is driven. For instance Shimamoto (1986) reported a transition between a localised ductile shear flow and a frictional slip.

Surprisingly, as far as we know, the constitutive description of these regime changes by phenomenological friction laws has received little attention (e.g. Heslot et al., 1994; Estrin and Bréchet, 1996; Lim and Chen, 1998). Nevertheless, the question of modelling different regimes of friction over a large span of slip rates is worthwhile. In addition to the constraints for the micromechanical modelling of friction carried by such a non-monotonic friction law, we show in this paper that the changes in behaviour of the friction coefficient with velocity determine the various and complex ways of accommodating a constant driving in the sliding interactions of two interfaces.

Starting with a short review of the rate-and-state concept of friction in the next section, we will propose a simple non-monotonic friction law for which the interfacial state variable remains bounded. After summarising the main results of the stability analysis of the sliding of a single interface, the dynamical system which determines the temporal behaviour of the two-interface problem is formulated. The bifurcation analysis of the two-interface steady states is then developed. This, finally, leads us to consider a modified version of the friction law initially proposed. An extensive bifurcation analysis of this modified friction law is carried out using continuation and direct time-integration methods.

\section{Rate-and-state friction}

The laws relating shear stress $\tau$ on an interface to the normal (compressive) stress $\sigma$ and the speed $v$ of sliding of the interface all have the general form (Rice et al., 2001)

$$
\left\{\begin{array}{l}
\tau=F(v, \psi, \sigma), \\
\dot{\psi}=-G(v, \psi, \sigma),
\end{array}\right.
$$

in which $\psi$ is a variable that describes the "state" of the interface, intended to reflect the complex interactions between the asperities on either side of the interface. The steady-state response of an interface sliding with speed $V$ is correspondingly given by

$$
\tau_{\mathrm{ss}}=F\left(V, \psi_{\mathrm{ss}}, \sigma\right),
$$


where $\psi_{\text {ss }}$ satisfies the equation

$$
G\left(V, \psi_{\mathrm{ss}}, \sigma\right)=0 .
$$

One particular realisation of the law (1) that was employed by Dieterich $(1978,1979)$ and Ruina $(1980,1983)$ is

$$
\left\{\begin{array}{l}
\tau=\left[a_{v}+a \ln \left(v / V_{*}\right)+b \ln \left(V_{*} \psi / L\right)\right] \sigma, \\
\dot{\psi}=1-v \psi / L .
\end{array}\right.
$$

This is commonly known as Dieterich's aging law (see Marone, 1998 for a review). Its fit to experimental data of Heslot et al. (1994) for steady-state sliding, obtained for Bristol paper board, is shown by the dashed line in Fig. 1(a), in which $\mu_{\mathrm{ss}}(V)$ denotes $\tau_{\mathrm{ss}} / \sigma$. It also fits qualitatively the observation that, if the sliding rate undergoes a step-function change, the friction law approaches its new steady-state value after the interface has slipped an amount of order L (Marone, 1998; Scholz, 1998, 2002). For present purposes, the most noteworthy drawback of the law is its logarithmic behaviour with sliding speed $v$, as $v \rightarrow 0$.

A "regularisation" of the Dieterich-Ruina law was proposed by Lapusta et al. (2000); see also Rice and Ben-Zion (1996), Ben-Zion and Rice (1997). They modified Eq. (4), expressed as a function $v(\tau, \psi)$, essentially by replacing the term $\exp (\tau / \sigma)$ by $2 \sinh (\tau / \sigma)$; this can be justified as allowing for backward jumps in the thermally activated process invoked for asperity creep (see Rice et al., 2001). The resulting law is

$$
\tau=a \sigma \sinh ^{-1}\left\{\frac{v}{2 V_{*}} \exp \left[\frac{a_{v}+b \ln \left(\psi / \psi_{*}\right)}{a}\right]\right\},
$$

where the reference state is $\psi_{*}=L / V_{*}$. When $a_{v} \gg a\left(a_{v} / a \sim 10\right.$ is sufficient $)$, this law still gives a monotonic steady-state response (decreasing in a manner which matches the data if $a<b)$ with $\tau_{\mathrm{ss}}\left(V_{*}\right) \approx a_{v} \sigma$ and hence an interfacial steady-state strength that increases indefinitely as $v \rightarrow 0$, if $a<b$. The source of this behaviour is the ability of the state variable $\psi$ to become indefinitely large. Therefore, we propose also to modify
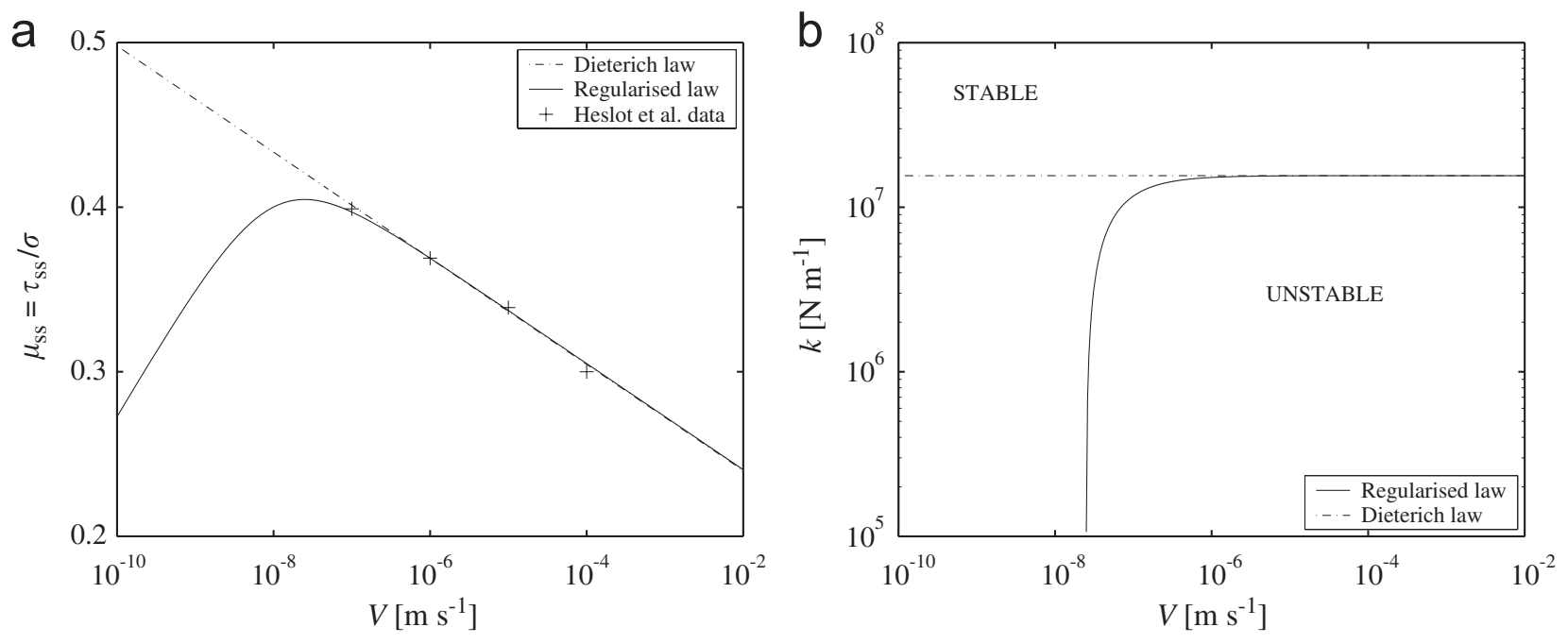

Fig. 1. Steady-state friction laws and stability domains: (a) the Dieterich-Ruina law is defined by Eq. (4) and its regularisation by (6); (b) corresponding critical stiffness $k_{\mathrm{c}}$ for the stick-slip instability onset. The following material parameters are used to fit the experimental results of Heslot et al. (1994): $a_{v}=0.369, a=0.0349$, $b=0.0489, \quad V_{*}=1 \mu \mathrm{m} \mathrm{s}^{-1}, \quad L=0.9 \mu \mathrm{m}$. For these parameter values $V_{\mathrm{M}}=2.49 \times 10^{-8} \mathrm{~m} \mathrm{~s}^{-1}$ and $\mu_{\mathrm{ss}}\left(V_{\mathrm{M}}\right)=0.405$. 
Eq. (4) $)_{2}$ to obtain

$$
\left\{\begin{array}{l}
\tau=a \sigma \sinh ^{-1}\left\{\frac{v}{2 V_{*}} \exp \left[\frac{a_{v}+b \ln \left(\psi / \psi_{*}\right)}{a}\right]\right\}, \\
\dot{\psi}=(1-\psi) / t_{* *}-|v| \psi / L
\end{array}\right.
$$

for which we define the reference state as $\psi_{*}=L /\left(L+V_{*} t_{* *}\right)$ so that Eq. (6) 1 is equivalent to Eq. (4) $)_{1}$ with $\tau_{\mathrm{ss}}\left(V_{*}\right) \approx a_{v} \sigma$ when $v>V_{*}$ and $a_{v} \gg a$. The second of these equations ensures that $\psi$ takes values between 0 and 1 . An evolution law for which $\psi$ remains bounded was previously proposed by Carlson and Batista (1996) in the context of a lubricated interface but with the feature that it leads to a steady-state friction law that is only piecewise-smooth.

More precisely, Eq. (6) 2 describes the evolution of the state due to two processes in competition: a (quasi-)stationary strengthening of the interface controlled by the time scale $t_{* *}$ and a dynamic weakening process on the time scale $L / V$. Our model has qualitative features in common with that proposed by Estrin and Bréchet (1996). They argue that continual healing as time increases should eventually saturate, as the effects of material hardening (not usually allowed for) become significant and suppress creep. They postulate that creep terminates once the asperity height reduces to a critical value $h_{0}$. Our time $t_{* *}$ would thus correspond to the time required for this to occur and should be externally controlled by the normal pressure, supposed fixed in this study. So, in the absence of interfacial movement, the interfacial state adjusts internally, by the creeping growth of asperity contact, towards $\psi=1$ which is the roughest possible state value. Now, considering the second process, it is interesting to see that the slip rate $V_{*}$ has no direct physical interpretation: it is a characteristic velocity selected arbitrarily as a reference for presenting experimental results. But, in many experimental papers it has been set to the value $V_{*} \approx 10^{-6} \mathrm{~ms}^{-1}$ and roughly coincides with the lower bound of the logarithmic velocity-weakening regime of friction. $L$ is the critical slip length over which the interface dynamically adjusts by the introduction or renewal of asperities, adapting the true area of interfacial contact, to any change in velocity. Therefore the timescale $t_{*} \equiv L / V_{*}$ is the upper bound of the characteristic timescales $L / V$ for this adjustment of the interfacial state for typical interfacial velocities. Intuitively it makes sense to take $t_{* *} \gg t_{*}$, since fluctuations in velocities that are of the order of $V_{*}$ or greater cause much more rapid adjustments in the interfacial state by rejuvenation of asperities than is possible in the quasi-stationary regime $\left(V \ll V_{*}\right)$ where the interfacial state evolves through slow creep of asperities.

As shown below, this competition of interfacial roughening/rejuvenation introduced in the state evolution law (6) 2 generates a non-monotonic steady-state friction law. A straightforward calculation using the steady-state form of (6) shows that the maximum of the frictional shear stress is attained at the slip rate

$$
V_{\mathrm{M}}=\frac{a L}{(b-a) t_{* *}} .
$$

We set $t_{* *} \gg t_{*} \equiv L / V_{*}$ so that $V_{\mathrm{M}} \ll V_{*}$. It follows that

$$
a_{v}<\frac{\tau_{\mathrm{ss}}\left(V_{\mathrm{M}}\right)}{\sigma}<\left[a_{v}-(b-a) \ln \left(\frac{b}{b-a} \frac{L / V_{*}}{t_{* *}}\right)\right]
$$


and slip velocities below $V_{\mathrm{M}}$ may be difficult to observe. The presence of such a local maximum in the steady-state behaviour of the dynamical friction coefficient should then be seen as a direct effect of the (quasi-)stationary interfacial healing process controlled by $t_{* *}$.

The existence of the maximum $\tau_{\mathrm{ss}}\left(V_{\mathrm{M}}\right)$ is indeed a consequence of the modified velocity dependence of the equilibrium value of the state

$$
\psi_{\mathrm{ss}}(V)=\frac{L / V}{L / V+t_{* *}}=\frac{L}{L+V t_{* *}},
$$

provided by Eq. (6) 2 . This expression suggests that, when the slip rate is very small, such that $V \ll L / t_{* *}$, the interface is rough and its state, close to 1 , does not vary with $V$. Consequently, the friction coefficient keeps its velocity-strengthening character contained in its unsteady form (6) $)_{1}$. But once the sliding is fast enough, larger than $L / t_{* *}$, the friction coefficient behaves in a velocity-weakening way as for the Dieterich-Ruina law and $\psi_{\mathrm{ss}} \approx(L / V) / t_{* *}$. An interpretation in terms of memory lengths is also possible by saying that the kinetic rejuvenation process of the interface, controlled by $L$, cannot take place when the sliding is so slow that $V t_{* *} \ll L$. As a result, the equilibrium value of the state $\psi_{\text {ss }}$ measures the efficiency of the dynamic rejuvenation mechanism due to sliding compared to the slow maturation of the interface in a quasi-stationary motion.

Fig. 1(a) shows the steady-state friction coefficients corresponding to the Dieterich-Ruina law and that described by Eq. (6). The values of the material parameters are those inferred from the experiment of Heslot et al. (1994). The characteristic time $t_{* *}$ is arbitrarily chosen to be $100 \times t_{*}$ but with the constraint of locating the local maximum of $\mu_{\mathrm{ss}}(V)$ for a slip rate $V_{\mathrm{M}}$ which is less than the velocities of the velocity-weakening region measured by Heslot et al. (1994). A further modification, which induces a change in behaviour at high slip rates, will be introduced in Section 4.

Finally we note that the expressions of the frictional shear stress $(4)_{1}$ or $(6)_{1}$ are very convenient for numerical purposes as they can easily be inverted to give the slip rate $v(\tau, \psi)$. They respectively lead to

$$
\begin{aligned}
& v=\exp \left(-a_{v} / a\right) V_{*}\left(\psi V_{*} / L\right)^{-b / a} \exp [\tau /(a \sigma)], \\
& v=2 \exp \left(-a_{v} / a\right) V_{*}\left(\psi / \psi_{*}\right)^{-b / a} \sinh [\tau /(a \sigma)] .
\end{aligned}
$$

These expressions could have been used to introduce rate-and-state friction according to the alternative view of Nakatani (2001) rather than the classical phenomenological viewpoint we recalled in this section.

\section{Steady states and their stability}

The remainder of this paper is concerned with a homogeneous elastic medium of thickness $H$, occupying the region $-\infty<x<\infty, 0<y<H,-\infty<z<\infty$. The lower boundary $y=0$ is held fixed, while the upper boundary $y=H$ moves with a constant horizontal velocity $V_{H}$, taken to be directed along $O x$. The medium is divided into layers whose interfaces are defined by $y=$ constant. Effects of elastic waves are ignored, and only slips that are uniform over any one interface are considered. It follows that the displacement components $(u, v)$ are linear functions of $y$ and independent of $x$; $v$ but not $u$ also depends on time $t$. Correspondingly, the normal stress $\sigma$ is constant and the shear stress $\tau$ is a function of $t$ only. 


\subsection{A single interface}

It is helpful for completeness, and to set the scene, to consider first a single horizontal interface which divides the medium into two; the exact position $y=h_{1}$ of the interface is unimportant when elastic waves are disregarded. Denote the interfacial slip (displacement in the $x$-direction) by $u_{1}(t)$ so that the corresponding slip velocity is $v_{1}(t)=\dot{u}_{1}(t)$. It follows that the $x$-displacement

$$
u(y, t)= \begin{cases}\tau(t) y / \mathrm{G} & \text { if } 0 \leqslant y<h_{1}, \\ \tau(t) y / \mathrm{G}+u_{1}(t) & \text { if } h_{1}<y \leqslant H,\end{cases}
$$

where $\mathrm{G}$ denotes the shear modulus of the medium. The condition at $y=H$ implies

$$
\dot{\tau}(t) / k+v_{1}(t)=V_{H},
$$

where $k \equiv \mathrm{G} / H$ denotes a "spring constant" for the medium. In the steady state, $\dot{\tau}=0$, $v_{1}=V_{H}$ and the steady-state friction law gives $\tau=\tau_{\mathrm{ss}}\left(V_{H}\right)$ and $\psi=\psi_{\mathrm{ss}}\left(V_{H}\right)$. Now consider a small perturbation of this state, so that $v_{1}=V_{H}+\delta v_{1}(t), \tau=\tau_{\mathrm{ss}}+\delta \tau(t)$ and $\psi=\psi_{\mathrm{ss}}+\delta \psi(t)$. It follows from the general friction law (1) that, to lowest order,

$$
\begin{aligned}
& \delta \tau=F_{V} \delta v_{1}+F_{\psi} \delta \psi, \\
& \delta \dot{\psi}=-G_{V} \delta v_{1}-G_{\psi} \delta \psi,
\end{aligned}
$$

where the function $F_{V}$ denotes the partial derivative $\partial F / \partial v$ evaluated at the steady state, and similarly for $F_{\psi}, G_{V}$ and $G_{\psi}$. The boundary condition (11) implies

$$
\delta \dot{\tau} / k+\delta v_{1}=0 .
$$

A non-trivial solution of Eqs. (12), (13) with time-dependence $e^{s t}$ is possible if $s$ satisfies

$$
F_{V} s^{2}+\left(k+G_{\psi} \mathrm{d} \tau_{\mathrm{ss}} / \mathrm{d} V\right) s+k G_{\psi}=0,
$$

where

$$
\mathrm{d} \tau_{\mathrm{ss}} / \mathrm{d} V=\left(F_{V} G_{\psi}-F_{\psi} G_{V}\right) / G_{\psi}
$$

is the slope of the steady-state friction law. It follows that steady-state sliding is stable if $k>-G_{\psi} \mathrm{d} \tau_{\mathrm{ss}} / \mathrm{d} V$, and hence for any $k$ if $\mathrm{d} \tau_{\mathrm{ss}} / \mathrm{d} V>0$, since $G_{\psi}>0$. If $\mathrm{d} \tau_{\mathrm{ss}} / \mathrm{d} V<0$, the steady state is stable so long as

$$
k>k_{\mathrm{c}} \equiv-G_{\psi} \mathrm{d} \tau_{\mathrm{ss}} / \mathrm{d} V .
$$

At $k=k_{\mathrm{c}}$, the system becomes unstable via a Hopf bifurcation with a frequency $\omega_{\mathrm{c}}$ given by $\omega_{\mathrm{c}}^{2}=G_{\psi} k_{\mathrm{c}} / F_{V}$. For the Dieterich-Ruina law (4), these values are

$$
k_{\mathrm{c}}=\sigma(b-a) / L, \quad \omega_{\mathrm{c}}^{2}=(b-a) / a .
$$

As discussed by Ruina (1983), Rice and Ruina (1983) and Gu et al. (1984), the Hopf bifurcation is indicative of stick-slip behaviour if $k<k_{\mathrm{c}}$. This contrasts with the law (6) for which $\mathrm{d} \tau_{\mathrm{ss}} / \mathrm{d} V>0$, for all $V<V_{\mathrm{M}}$, and correspondingly the steady state is stable for all $k$. Fig. 1(b) shows the domains of stability and instability in the $(k, V)$ plane for each of these laws. 


\subsection{Two interfaces}

\subsubsection{Two steady states}

Dividing the medium into three with a supplementary interface introduces an additional slip rate $v_{2}(t)$ in the boundary condition (11) which then becomes

$$
\dot{\tau}(t) / k+v_{1}(t)+v_{2}(t)=V_{H} .
$$

Assuming the same constitutive frictional behaviour of the two interfaces, Eq. (18) together with (1) gives us the dynamical system which determines the temporal behaviour of the two-interface system

$$
\begin{aligned}
& \dot{\tau}=k\left[V_{H}-v_{1}\left(\tau, \psi_{1}\right)-v_{2}\left(\tau, \psi_{2}\right)\right], \\
& \dot{\psi}_{1}=-g\left(\tau, \psi_{1}\right), \\
& \dot{\psi}_{2}=-g\left(\tau, \psi_{2}\right)
\end{aligned}
$$

in which the functions $v_{i}\left(\tau, \psi_{i}\right)$ and $g\left(\tau, \psi_{i}\right)$ are obtained by inversion of Eq. $(1)_{1}$ and combination with $G(v, \psi)$.

The two-interface model contains a symmetry in that $\psi_{1}$ and $\psi_{2}$ may be interchanged without altering the ODEs (19). A consequence is the existence of a flow-invariant subspace for the dynamics in which $\psi_{1}=\psi_{2}$. In what follows we will refer to solutions for which $\psi_{1}(t)=\psi_{2}(t)$ as "symmetric" and solutions for which $\psi_{1}(t) \neq \psi_{2}(t)$ as "asymmetric." This important remark will be useful to understand qualitatively the trajectories of the system later on. For now, we can bear in mind that the symmetric solutions behave like the solutions of the single-interface problem. Steady states will be referred to as equilibria, as in the standard vocabulary of dynamical systems theory.

In steady state, according to Eq. (19) $)_{1}$, the driving velocity $V_{H}$ is distributed over the two interfaces while the slip rates of the two interfaces $v_{1}(t) \equiv V_{1}$ and $v_{2}(t) \equiv V_{2}$ are related by the steady-state friction law (2) because the shear stress is constant and uniform in the medium. The steady-state slidings then are solutions of the nonlinear system of equations

$$
\left\{\begin{array}{c}
V_{1}+V_{2}=V_{H}, \\
\tau_{\mathrm{ss}}\left(V_{1}\right)=\tau_{\mathrm{ss}}\left(V_{2}\right) .
\end{array}\right.
$$

As for the single interface case, the steady states are independent of the stiffness of the medium but parametrised by $V_{H}$. The stationary state $V_{1}=V_{2}=V_{H} / 2$ is the obvious symmetric solution of Eq. (20) and is unique if the steady-state friction law is a monotonic function of $V$. But, more interestingly, and because the shear stress must be constant in steady-state, an asymmetric solution $V_{1} \neq V_{2}$ is expected for a non-monotonic steady-state friction law. Moreover, by analogy with the single-interface problem, we expect the symmetric solution to be stable (resp., unstable) if $V_{H} / 2$ is associated with a velocity strengthening (resp., weakening) part of the steady-state friction law. This suggests that the symmetric solution might bifurcate to the asymmetric solution when $V_{H} / 2$ reaches the slip rate $V_{\mathrm{e}}$ at which the shear stress is extremum. As a consequence, by reason of symmetry, a pitchfork bifurcation is expected at $V_{H}=2 V_{\mathrm{e}}$. A local analysis in the vicinity of $2 V_{\mathrm{e}}$ confirms this intuition.

Indeed, let us consider the bifurcation theory of equilibrium solutions as a consequence of the implicit function theorem (e.g. Iooss and Joseph, 1980) in order to characterise the 
existence and uniqueness of the solutions of Eq. (20). First, let us rewrite Eq. (20) as a single equation by using the constraint $(20)_{1}$ which implies that the interfaces slide with the slip rates

$$
V_{1}=\frac{V_{H}}{2}+\frac{V_{1}-V_{2}}{2} \text { and } \quad V_{2}=\frac{V_{H}}{2}-\frac{V_{1}-V_{2}}{2} .
$$

Letting $\lambda=V_{H} / 2$ and $z=\left(V_{1}-V_{2}\right) / 2$, the steady-state slidings of the system are solutions of Eq. $(20)_{2}$ rewritten as

$$
\phi(z ; \lambda)=\tau_{\mathrm{ss}}(\lambda+z)-\tau_{\mathrm{ss}}(\lambda-z)=0 .
$$

So, from the implicit function theorem, a unique solution $z(\lambda) \neq 0$ of the latter equation exists if $\phi_{z} \equiv \partial \phi / \partial z \neq 0$. If

$$
\phi_{z}=\tau_{\mathrm{ss}}^{\prime}(\lambda+z)+\tau_{\mathrm{ss}}^{\prime}(\lambda-z)=0,
$$

there is a singular point $\left(z_{\mathrm{c}}, \lambda_{\mathrm{c}}\right)$ which satisfies Eqs. (21) and (22). Considering the symmetric solution $z=0$, the condition (22) immediately shows that it bifurcates for a value $\lambda_{\mathrm{c}}=V_{\mathrm{e}}$ corresponding to an extremum of $\tau_{\mathrm{ss}}$. Thus, there exists a critical value of the driving velocity

$$
V_{H}^{\mathrm{c}}=2 V_{\mathrm{e}},
$$

from which the asymmetric solution appears. Indeed, a local analysis around $\lambda_{\mathrm{c}}$ shows that the solution $z=0$ bifurcates to the solution $z \neq 0$ by a pitchfork bifurcation. From a third order Taylor expansion of $\phi(z, \lambda)$ with $z=z_{\mathrm{c}}+\varepsilon z_{1}+\varepsilon^{2} z_{2}+\cdots$ and $\lambda=\lambda_{\mathrm{c}}+\varepsilon \lambda_{1}+$ $\varepsilon^{2} \lambda_{2}+\cdots$, it is found that $\lambda_{1}=0$ and

$$
z_{1}^{2}=-6\left[\tau_{\mathrm{ss}}^{\prime \prime}\left(\lambda_{\mathrm{c}}\right) / \tau_{\mathrm{ss}}^{\prime \prime \prime}\left(\lambda_{\mathrm{c}}\right)\right] \lambda_{2}
$$

Depending on the shape of the steady-state friction law about the extremum, the previous expression shows that the nature of the pitchfork bifurcation is either supercritical or subcritical if we assume that the solution $V_{1}=V_{2}$ is stable for $V_{H} / 2$ belonging to the strengthening part of the steady-state friction law. This latter hypothesis is shown to be correct in the next section. Indeed, let us assume for instance that the steady-state friction law has a maximum at $V_{\mathrm{M}}$. Because then $\tau_{\mathrm{ss}}^{\prime \prime}\left(V_{\mathrm{M}}\right)<0$, the solution $z \neq 0$ appears for $V_{H}>2 V_{\mathrm{M}}$ from a supercritical pitchfork bifurcation if the steady-state friction law has a velocity strengthening branch which is steeper than the weakening one, i.e. $\tau_{\mathrm{ss}}^{\prime \prime \prime}\left(V_{\mathrm{M}}\right)>0$. Then,

$$
z \simeq \varepsilon z_{1}= \pm \sqrt{-3 \tau_{\mathrm{ss}}^{\prime \prime}\left(V_{\mathrm{M}}\right) / \tau_{\mathrm{ss}}^{\prime \prime \prime}\left(V_{\mathrm{M}}\right)} \sqrt{V_{H}-2 V_{\mathrm{M}}}
$$

For a steady-state friction law with a local minimum at $V_{H}=2 V_{\mathrm{m}}$, similar reasoning applies. The different possible cases are summarised in Fig. 2.

In summary, the nature of the pitchfork bifurcation depends on the symmetry of the steady-state friction law about the extremum. To exist, the asymmetric solution $V_{1} \neq V_{2}$ requires a steady-state friction law which is not symmetric about a local extremum and with a velocity strengthening part steeper than the weakening one.

\subsubsection{Linear stability analysis}

The response of the symmetric and asymmetric steady-states to a small perturbation is studied as explained in Section 3.1. The growth rates of perturbations are given by the 


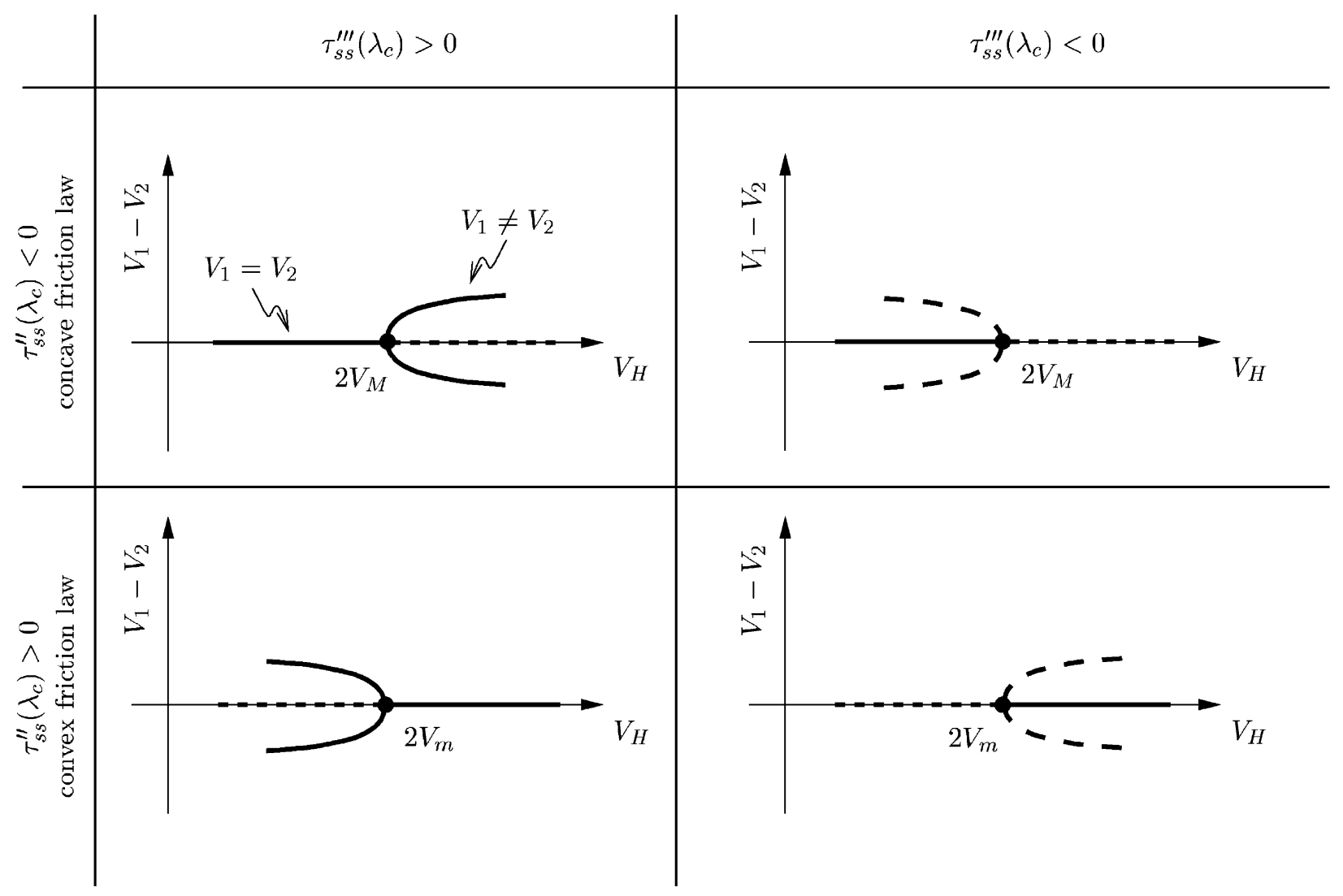

Fig. 2. Pitchfork bifurcation of the steady-state slidings of the two-interface system: the shape of the steady-state friction law controls the existence of the solutions of Eqs. (20). $V_{\mathrm{M}}$ is a local maximum in $\tau_{\mathrm{ss}}, V_{\mathrm{m}}$ is a local minimum in $\tau_{\mathrm{ss}}$.

eigenvalues of the Jacobian matrix of Eq. (20) which is written as follows:

$$
\left(\begin{array}{ccc}
-k\left(1 / F_{V}^{(1)}+1 / F_{V}^{(2)}\right) & k F_{\psi}^{(1)} / F_{V}^{(1)} & k F_{\psi}^{(2)} / F_{V}^{(2)} \\
-G_{V}^{(1)} / F_{V}^{(1)} & k_{\mathrm{c}}^{(1)} / F_{V}^{(1)} & 0 \\
-G_{V}^{(2)} / F_{V}^{(2)} & 0 & k_{\mathrm{c}}^{(2)} / F_{V}^{(2)}
\end{array}\right)
$$

in terms of the functions $F$ and $G$ and where

$$
k_{\mathrm{c}}^{(i)}=-G_{\psi}^{(i)} \tau_{\mathrm{ss}}^{\prime}\left(V_{i}\right)
$$

The superscripts ${ }^{(1)}$ and ${ }^{(2)}$ denote the two interfaces and mean that the partial derivatives of $F$ and $G$ are evaluated at $V_{1}$ or $V_{2}$.

About the symmetric solution, the characteristic equation of the Jacobian reduces to

$$
\left[s^{2}-s\left(k_{\mathrm{c}}-2 k\right) / F_{V}+2 k G_{\psi} / F_{V}\right]\left(k_{\mathrm{c}} / F_{V}-s\right)=0 .
$$

The quadratic factor in this polynomial is denoted $P_{2}$ in the following. The three roots of (26) are

$$
\begin{aligned}
& s=k_{\mathrm{c}} / F_{V} \\
& s_{ \pm}=\frac{k_{\mathrm{c}}-2 k}{2 F_{V}} \pm \frac{\left[\left(k_{\mathrm{c}}-2 k\right)^{2}-8 k G_{\psi} F_{V}\right]^{1 / 2}}{2 F_{V}} .
\end{aligned}
$$


It is easy to show geometrically that the three roots have negative real parts when $k_{\mathrm{c}}<0$, that is when $V_{H} / 2$ is in the range of slip rate where there is a velocity strengthening of the steady-state friction law. On the other hand, when $k_{\mathrm{c}}>0$, the symmetric solution is always unstable whatever the value of $k$ because of the root $k_{\mathrm{c}} / F_{V}$. This eigenvalue corresponds to the stationary pitchfork bifurcation we described earlier. Meanwhile, as we will discuss in Section 4.2, the two other roots $s_{ \pm}$are important for understanding the bifurcation diagram of the dynamical system (19) as they define a Hopf bifurcation on the unstable part of the branch of the symmetric solution. Their real parts vanish for a critical stiffness

$$
k_{\mathrm{c}}^{s}=\frac{k_{\mathrm{c}}\left(V_{H} / 2\right)}{2} \text {. }
$$

The stability of the asymmetric solution is determined by the roots of the cubic equation

$$
P_{3}(s)=-\left(s_{3}+s\right)\left(s-s_{1}\right)\left(s-s_{2}\right)-k a_{2} s_{2}\left(s-s_{1}\right)-k a_{1} s_{1}\left(s-s_{2}\right),
$$

where we define

$$
\begin{aligned}
& a_{1} s_{1} \equiv F_{\psi}^{(1)} G_{V}^{(1)} / F_{V}^{(1) 2}=\left(1 / F_{V}^{(1)}-1 / \tau_{V}^{(1)}\right) s_{1}, \\
& a_{2} s_{2} \equiv F_{\psi}^{(2)} G_{V}^{(2)} / F_{V}^{(2) 2}=\left(1 / F_{V}^{(2)}-1 / \tau_{V}^{(2)}\right) s_{2}
\end{aligned}
$$

and

$$
s_{3} \equiv k a_{3}=k\left(1 / F_{V}^{(1)}+1 / F_{V}^{(2)}\right) .
$$

With no loss of generality as the indices 1 and 2 play a symmetrical role, let us here assume that $s_{1}>0$ and $s_{2}<0$. In this way, we consider that $V_{1}$ is associated to the velocity weakening part of the steady-state friction law. In addition, to find a criterion of stability, let us consider that the slip rates $V_{1}$ and $V_{2}$ are such that $s_{1}+s_{2}<0$. It is reasonable to make this assumption as we have seen before that $V_{1}$ and $V_{2}$ are not very different from $V_{H} / 2$ in the neighbourhood of $V_{H}^{\mathrm{c}}$. Therefore, we can suppose that the term $G_{\psi}^{(i)} / F_{V}^{(i)}$ in the definition of $s_{i}$ can be approximated by its value taken at $V_{H} / 2$. Thus, the sum $s_{1}+s_{2}$ has the sign of the $s_{i}$ whose index corresponds to the slip rate of the largest derivative $\tau_{\mathrm{ss}}^{\prime}\left(V_{i}\right)$ in absolute value. Considering that the strengthening is faster than the velocity weakening, we obviously have $\left|s_{2}\right|>s_{1}>0$ and then $s_{1}+s_{2}<0$. The stability of $P_{3}$ is analysed for this case in Appendix A. It is found that the asymmetric solution becomes unstable at a Hopf bifurcation if $k$ is less than a critical stiffness given by

$$
k_{\mathrm{c}}^{*}=\frac{1}{2} \frac{s_{1}^{2}\left(a_{1}-a_{3}\right)+s_{2}^{2}\left(a_{2}-a_{3}\right)-2 s_{1} s_{2} a_{3}+\sqrt{\Delta}}{a_{3}\left[s_{1}\left(a_{1}-a_{3}\right)+s_{2}\left(a_{2}-a_{3}\right)\right]} .
$$

The expression of $\Delta$ is given by Eq. (43) of Appendix A. At the onset of instability we find the oscillation frequency to be

$$
\omega_{*}^{2}=\frac{-s_{1} s_{2}\left(a_{1}+a_{2}-a_{3}\right) k_{\mathrm{c}}^{*}}{-\left(s_{1}+s_{2}-k_{\mathrm{c}}^{*} a_{3}\right)} .
$$

\subsection{A numerical illustration}

The preceding analysis shows that the sliding behaviour of the two interfaces strongly depends on the monotonicity of the steady-state friction law and leads to a selection of the active interfaces. Before giving a more complete picture of the behaviour of the 
two-interface system from a numerical continuation method, we illustrate the results of the previous analysis by solving numerically the dynamical system (19) for the Dieterich-Ruina law (8). We verify that the two-interface system is unstable when a monotonic velocity-weakening friction law is considered. To do so, a direct numerical integration of the dimensionless form of Eqs. (19) and (8), obtained by considering the characteristic time $L / V_{*}$, length $L$ and stress $a \sigma$, is performed using the Matlab routine ode23s. The parameters $k$ and $V_{H}$ being chosen, the integration starts at an initial condition $\left[\tau(0), \psi_{1}(0)\right.$, $\left.\psi_{2}(0)\right]$ close to the steady-state symmetric solution $\left[\tau_{\mathrm{ss}}\left(V_{H} / 2\right), \psi_{1}^{\mathrm{ss}}\left(V_{H} / 2\right), \psi_{2}^{\mathrm{ss}}\left(V_{H} / 2\right)\right]$. The trajectory so generated indicates stability of the steady state if it converges to it, or instability otherwise.

Fig. 3 compares, for $V_{H}=V_{*}$ and two different values of the dimensionless stiffness $K=L k /(a \sigma)$, the evolution of the system projected onto the two-dimensional plane $\left(\tau, v_{1}-v_{2}\right)$, the time evolution of $\tau, \psi$ and $v$. Fig. 3(a) shows the localisation of the slip
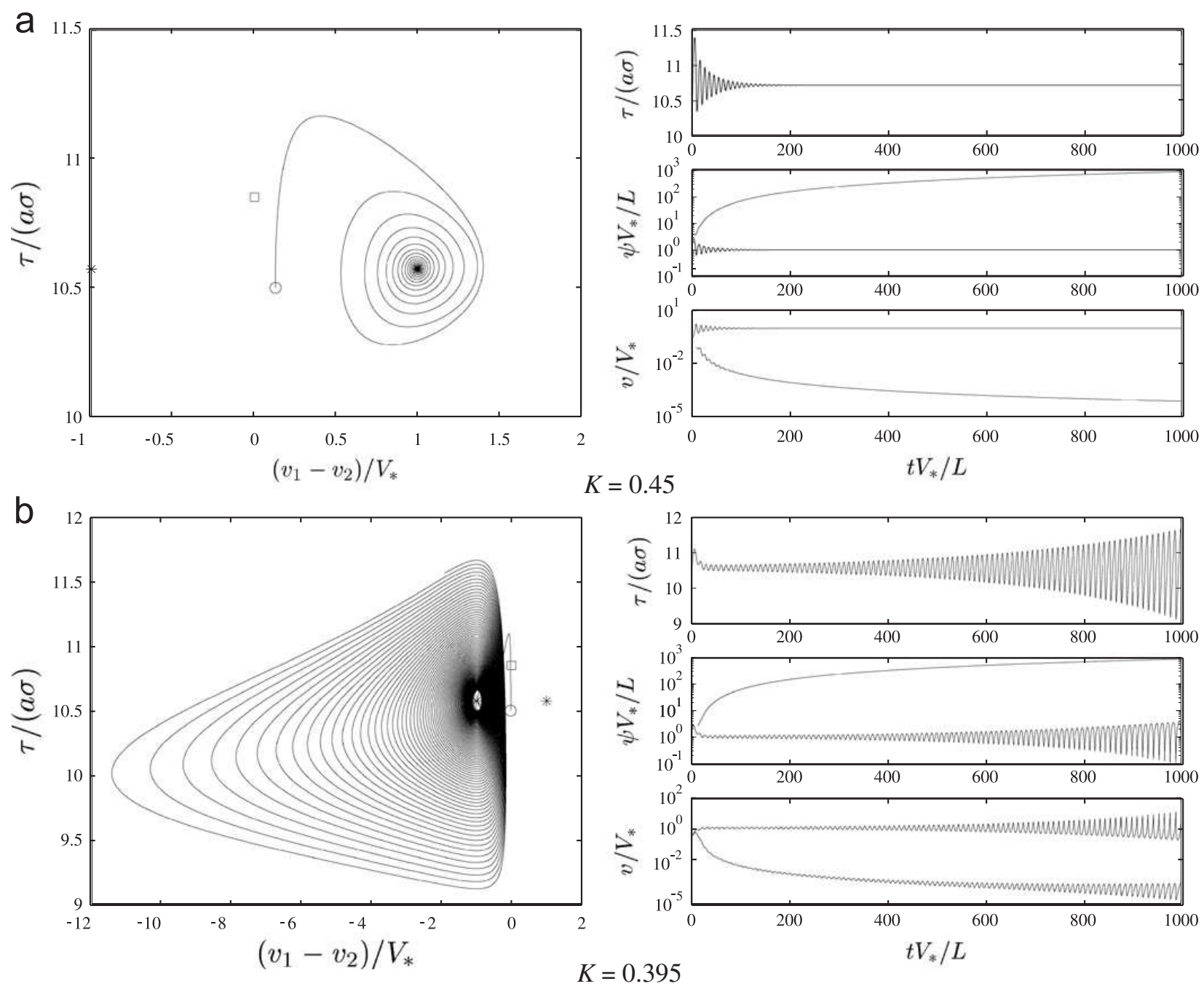

Fig. 3. Numerical solutions of Eq. (19) for the velocity-weakening friction law of Dieterich-Ruina (4): the twointerface system is driven at $V_{H}=V_{*}$ and shows the selection of the weakest interface. (a) The symmetric steady state $V_{1}=V_{2}=V_{H} / 2$ is unstable. (b) A stick-slip oscillation develops on the selected active interface if the stiffness is less than the critical value of the single interface problem (cf. Eq. (17)). In the phase space, the symbols $\circ, \square, *$, respectively, correspond to the initial condition, the symmetric solution $V_{1}=V_{2}=V_{H} / 2$, and the two possible steady states of the single interface problem. In both (a) and (b) steady states $*$ are located at $(1,10.7)$ and $(-1,10.7)$. 
along one interface in the sense that one of the two interfaces slows down by several orders of magnitude, the other one speeding up. In the phase space, it means that the trajectory goes towards the single-interface steady state of the speeding-up interface. The selected slipping interface is the one which had the lower state variable as initial condition, that is the weaker interface. Ultimately, the system behaves as if the more resistive interface had stopped. Indeed, the state variable of the slower interface rises which increases the resistance of this interface to its motion. Therefore, its slip rate reduces because the shear stress has to remain constant. The converse happens for the accelerating interface.

We can check numerically that the stiffness has no effect on the stability of the twointerface system in the present situation. However, depending on the value of the stiffness $K$ compared to its critical value, here $K_{\mathrm{c}}=(b-a) / a \approx 0.401$, which is given by the linear stability analysis of the single-interface problem, a "stick-slip" instability can appear along the selected interface. Fig. 3(b) shows the growth of this instability close to its onset. The stick-slip on the active interface produces shear stress oscillations. Consequently, the slow interface, which has almost stopped, also oscillates. The Dieterich-Ruina friction law does not allow the slow interface to accelerate again though; the state variable has reached too large a value.

Whereas during slip of a single interface the possibility of infinite hardening associated with the Dieterich state evolution law $(4)_{2}$ is not an issue, this feature is exposed when two interfaces are considered; some modification such as that embodied in Eq. (6) 2 becomes desirable. However, the fact that, when a monotonic velocity-weakening friction law is supposed, the two-interface system (as for $N$ interfaces, see Putelat and Willis, 2004) results in localisation of the slip along one interface, justifies and emphasises the importance of all the work done by many authors in recent years concerning the single interface problem.

\section{A regularised two-interface model}

\subsection{A spinodal friction law}

Inspection of the expressions (6) $)_{1}$ or (9) shows that these equations become singular at large velocities when $\psi \rightarrow 0$ if we wish to keep the shear stress finite. Therefore, we propose to modify Eq. (6) $)_{1}$ by introducing a small constant $c$ whose physical significance is to give the interface a residual strength even at very high slip rates for which $\psi \approx 0$

$$
\tau=a \sigma \sinh ^{-1}\left[\frac{v}{2 V_{*} \exp \left(-a_{v} / a\right)}\left(c+\frac{\psi}{\psi_{*}}\right)^{b / a}\right] .
$$

As a consequence, a velocity-strengthening regime appears for high speeds. Note that the constant $c$ is related to the transition velocity $V_{T}\left(=V_{*} / c\right)$ proposed by Weeks (1993) in logarithmic friction laws to introduce high-velocity strengthening. Provided heating and melting are disregarded (Rice, 2006), such transition from weakening to strengthening for moderately high velocities might be a universal feature of friction as experimentally observed in different materials (e.g. for rocks, Weeks and Tullis, 1985; Shimamoto, 1986; Kilgore et al., 1993; Heslot et al., 1994; Rabinowicz, 1995, for paper and metals). The state variable $\psi_{*}=\left(1+t_{* *} V_{*} / L\right)^{-1}$ corresponds to the slip rate of reference $V_{*}$ and has been introduced in the definition of the friction law so that the coefficient $a_{v}$ is approximately the value of the friction coefficient for the slip rate $V_{*}$. 
Considering the characteristic time $L / V_{*}$ and length $L$ ( $\mathrm{Gu}$ et al., 1984), the dimensionless modified version of (6) is

$$
\left\{\begin{array}{l}
\tau=\sinh ^{-1}\left\{v[c+(1+R) \psi]^{\beta} / \gamma_{0}\right\}, \\
\dot{\psi}=(1-\psi) / R-|v| \psi,
\end{array}\right.
$$

in which we define $\beta=b / a, R=t_{* *} V_{*} / L$ and $\gamma_{0}=2 \exp \left(-a_{v} / a\right)$. The corresponding steady-state friction law

$$
\tau_{\mathrm{ss}}=\sinh ^{-1}\left[\frac{v}{\gamma_{0}}\left(c+\frac{1+R}{1+R v}\right)^{\beta}\right],
$$

has a local maximum and a local minimum for the respective slip rates

$$
\frac{V_{\mathrm{M}}}{V_{*}} \approx \frac{1}{R(\beta-1)} \text { and } \quad \frac{V_{\mathrm{m}}}{V_{*}} \approx \frac{1+R}{R} \frac{\beta-1}{c} .
$$

The latter expression shows that $V_{\mathrm{m}} \rightarrow \infty$ as $c \rightarrow 0$, the law (6) becoming a limiting case of Eq. (35). We take the value of $c$ to be fixed at $10^{-3}$ so that the experimental data of Heslot et al. (1994) are well fitted, see Fig. 4(a). Consequently, the extrema of $\tau_{\mathrm{ss}}$ are reached for

$$
V_{\mathrm{M}} / V_{*} \approx 0.025 \text { and } V_{\mathrm{m}} / V_{*} \approx 405.16
$$

By analogy with various other physical phenomena, for example the isotherms of the pressure-density relation of the van der Waals fluid, and the separation of a solidifying binary alloy, we describe for brevity such a non-monotonic friction law as "spinodal." For a single interface, the existence of the local maximum and minimum of $\tau_{\mathrm{ss}}$ closes the region of instability in the $(V, k)$ plane where stick-slip oscillations exist, see Fig. 4(a). When two interfaces are considered, this feature produces interesting interactions between the interfaces, although the most important effects are related to the existence of local extrema and not the global spinodal shape of the friction law.

a

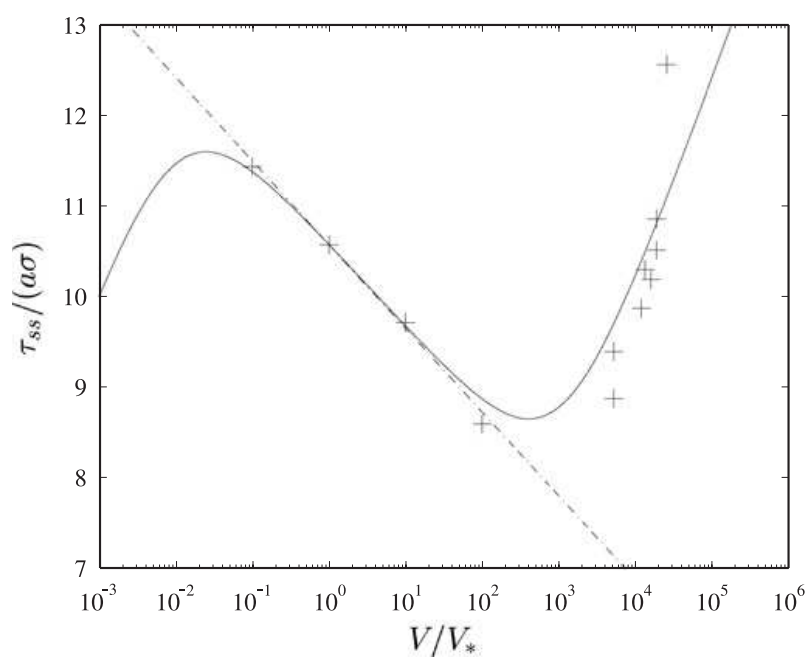

b

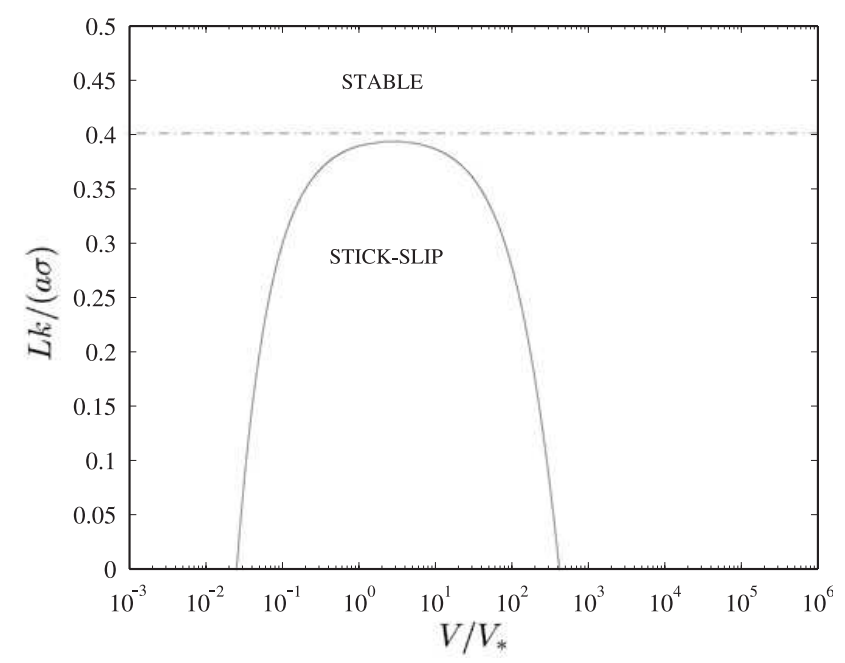

Fig. 4. Spinodal friction law (35) and stability domain of the single interface problem. Symbols + refer to the experimental data of Heslot et al. (1994) and the dotted-dashed line - - - - - to the Dieterich-Ruina friction law (4). 


\subsection{Bifurcations and regime diagrams}

The system of three nonlinear ODEs (19) with the modified friction law (35) was investigated in detail using the continuation package Auto (Doedel et al., 1991). The occurrence in the ODEs of several constants that are fixed to take either very large or very small values (e.g. $R$ and $c$ ) makes numerical examination of the problem slightly awkward. In particular, we found that numerical difficulties following periodic orbits arose because $\psi_{1}$ and $\psi_{2}$ routinely became very small. This was remedied by defining the new variables $w_{j}=\log _{10}\left(\psi_{j}\right)$ and performing continuation with the $\left(\tau, w_{1}, w_{2}\right)$ system.

A general outline of the dynamics is shown in Figs. 5-7 which nicely illustrate the analysis developed in Section 3.2.1. A branch of symmetric equilibria, with a characteristic spinodal shape following from the constitutive relation $\tau_{\mathrm{ss}}\left(V_{H} / 2\right)$ exists over the entire interval of $V_{H}$ considered: $10^{-3} \leqslant V_{H} / V_{*} \leqslant 10^{6}$, see Fig. 5(a). The symmetric equilibrium is stable for $V_{H}$ at the ends of this interval, and loses stability at pitchfork bifurcations at $V_{H}=2 V_{\mathrm{m}}$ and $V_{H}=2 V_{\mathrm{M}}$ near the extrema of the constitutive relation curve, as found analytically in Section 3.2.1. Note finally that these equilibria and the locations of their bifurcation points are independent of the parameter $K$ as the form of the ODEs clearly demonstrates.

Interestingly, while the pitchfork bifurcation at $V_{H}=2 V_{\mathrm{M}}$ is supercritical, that at $V_{H}=$ $2 V_{\mathrm{m}}$ is subcritical (cf. Section 3.2.1) and generates a pair of unstable equilibria that exist for $2 V_{\mathrm{m}} \leqslant V_{H} \leqslant V_{\mathrm{sn}} \approx 4.30 \times 10^{4} V_{*}$. Exactly, $\tau_{\mathrm{ss}}\left(V_{\mathrm{sn}}\right)=\tau_{\mathrm{ss}}\left(V_{\mathrm{M}}\right)$. At $V_{H}=V_{\text {sn }}$ the pair of unstable asymmetric equilibria collide in a saddle-node bifurcation with the pair of stable asymmetric equilibria created in the supercritical pitchfork bifurcation at $V=2 V_{\mathrm{M}}$, and thus disappear. Physically, this behaviour of the asymmetric equilibrium is a consequence of the spinodal shape of the friction law: a slow interface can only exist if $\tau_{\mathrm{ss}}\left(V_{\mathrm{m}}\right) \leqslant \tau_{\mathrm{ss}} \leqslant \tau_{\mathrm{ss}}\left(V_{\mathrm{M}}\right)$. As a result, the saddle-node bifurcation is reached (cf. Fig. 5(a)) at a slip rate such that

$$
\left.\tau_{\mathrm{ss}}\left(V_{\mathrm{sn}}\right) \approx \tau\left[V_{\mathrm{sn}}, \psi_{\mathrm{ss}}\left(V_{\mathrm{sn}}\right)=0\right] \approx \tau_{\mathrm{ss}}\left(V_{\mathrm{M}}\right)\right|_{c=0}
$$

which provides the approximate solution

$$
\frac{V_{\mathrm{sn}}}{V_{*}} \approx \frac{(\beta-1)^{\beta-1}}{R}\left(\frac{1+R}{c \beta}\right)^{\beta} \approx 4.440 \times 10^{4} .
$$

The asymmetric equilibrium solution cannot exist when the driving velocity $V_{H}$ is associated to a shear stress larger than $\tau_{\mathrm{ss}}\left(V_{\mathrm{M}}\right)$, characterised by having one interface moving slowly, at $V<V_{\mathrm{M}}$, as illustrated in Fig. 5(d). Thus, for driving velocities such that $V_{\mathrm{M}} \ll V<V_{\text {sn }}$, steady states in the two-interface system behave as if only one interface were present and the shear stress follows the steady-state friction law (cf. Fig. 5(a)).

For small enough $K$, Hopf bifurcations are possible from both the symmetric and the asymmetric equilibria. The locations of these Hopf bifurcations may be found in a similar way to Section 3.2.2 where we determine the critical stiffnesses (29) and (34) for which the equilibria become linearly unstable. The Hopf bifurcation curves, computed by a twoparameter continuation in $K$ and $V_{H}$, are shown in Fig. 6. For small $K$ it is found that both Hopf bifurcation curves approach the pitchfork bifurcation curve at $V=2 V_{\mathrm{M}}$. This feature is very important for the mechanical behaviour of the two-interface system, because it allows an interaction between the Hopf bifurcations and the pitchfork bifurcation, leading to complex dynamics. This is detailed in the next section. On the 
a

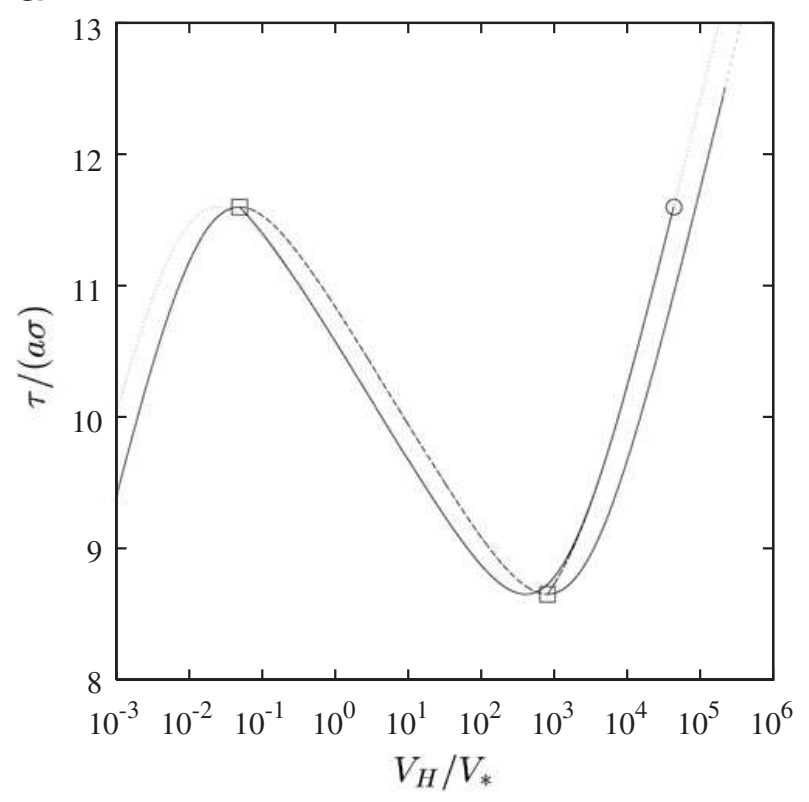

C

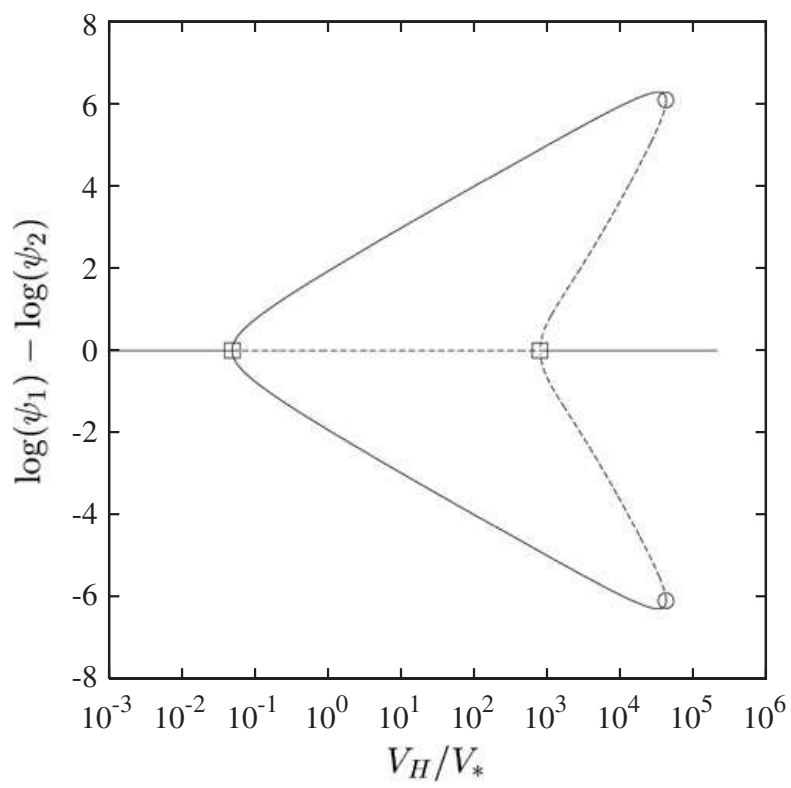

b

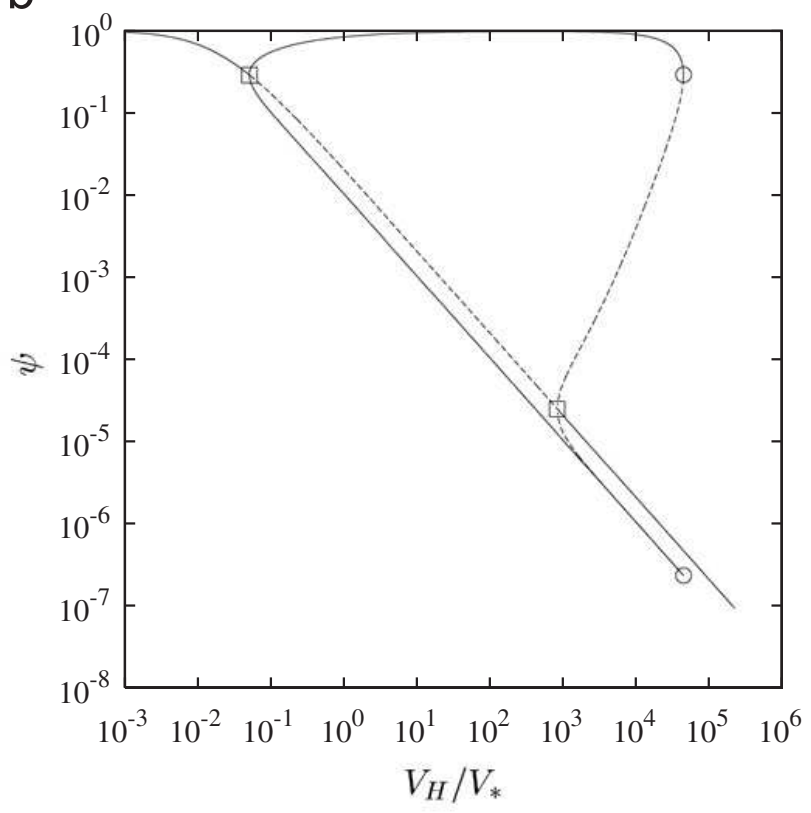

d

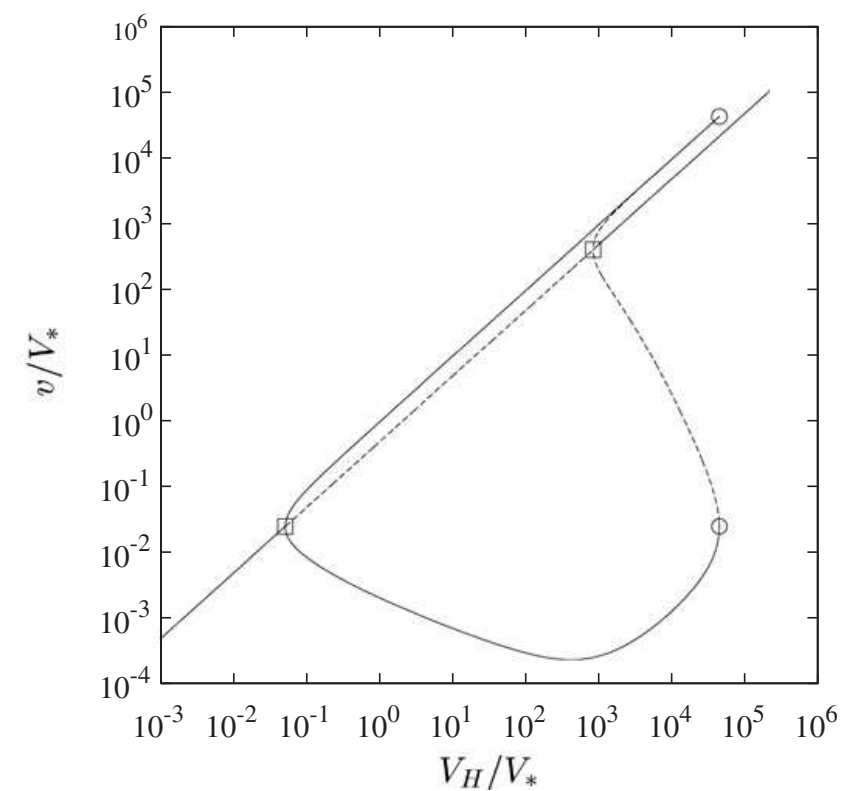

Fig. 5. Bifurcation diagrams of steady states for $K=0.45$. The symbol $\square$ denotes the pitchfork bifurcation of asymmetric steady states from the symmetric one. The symbol $\circ$ denotes a saddle-node bifurcation on the branch of asymmetric solutions. Solid lines — and dashed lines - - - symbolise stable and unstable steady states. In (a), the thin dotted and dashed lines, respectively, are the curves $\tau_{\mathrm{ss}}\left(V_{H} / V_{*}\right)$ and $\tau_{\mathrm{ss}}\left(V_{H} /\left(2 V_{*}\right)\right)$ calculated from the steady-state friction law (35).

contrary, such an interaction between bifurcation points does not exist for slip rates larger than $V_{\mathrm{m}}$ because of the subcritical nature of the pitchfork bifurcation at $V_{H}=2 V_{\mathrm{m}}$ which makes the stable symmetric and asymmetric equilibria widely separated. Because the asymmetric equilibrium is equivalent to the single interface system, its Hopf bifurcation curve behaves like the $k_{\mathrm{c}}(V)$ given by Eq. (16) for the single interface and vanishes at $V_{H}=V_{\mathrm{m}}$. This prevents any interaction with the pitchfork bifurcation at $V_{H}=2 V_{\mathrm{m}}$.

The Hopf bifurcations from the asymmetric equilibria are numerically found always to be supercritical; stable periodic oscillations exist everywhere below the dot-dashed curve in 


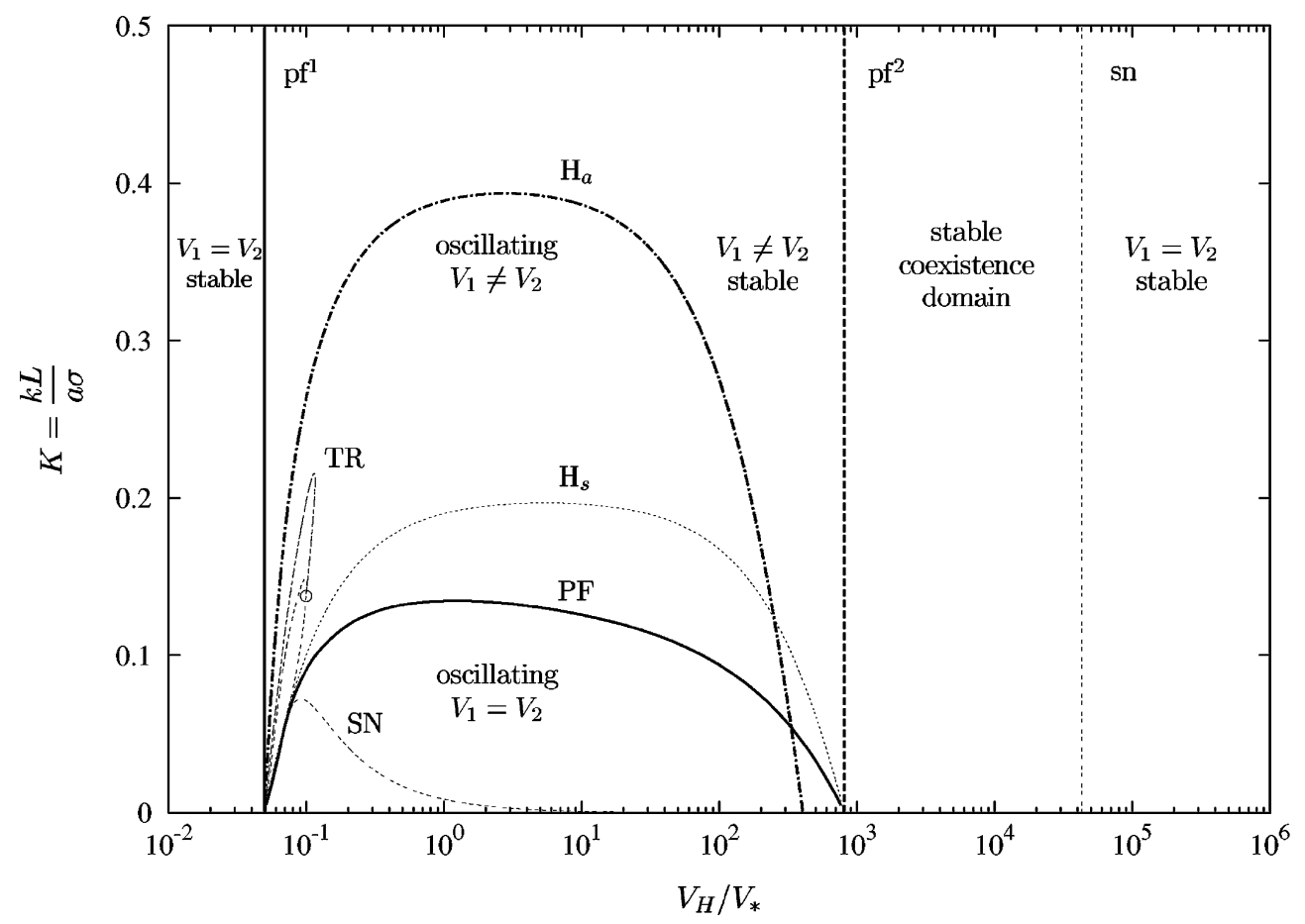

Fig. 6. Phase diagram of the two-interface system: — locus $V_{H}=2 V_{\mathrm{M}}$ of the supercritical pitchfork bifurcation $\left(\mathrm{pf}^{1}\right)$ of the symmetric stationary solution $V_{1}=V_{2}$ to the asymmetric stationary solution $V_{1} \neq V_{2}$, - - - locus $V_{H}=2 V_{\mathrm{m}}$ of the subcritical pitchfork bifurcation $\left(\mathrm{pf}^{2}\right)$, - - - locus $V_{H}=V_{\text {sn }}$ of the saddle-node bifurcation (sn) of the asymmetric solution, - - - - - locus of the saddle-node bifurcation (SN) of the asymmetric oscillatory solution, - - - - locus of the Hopf bifurcation $\left(\mathrm{H}_{\mathrm{a}}\right)$ of the asymmetric stationary solution, .... locus of the Hopf bifurcation $\left(\mathrm{H}_{\mathrm{s}}\right)$ of the unstable symmetric solution $V_{1}=V_{2}$. Below the thick four-dotted line (PF), symmetric oscillatory solutions can coexist with the asymmetric ones. The thin dashed-dotted and three-dotted lines, respectively, are the locus of the torus (TR) and period-doubling bifurcations of asymmetric solution. Complex dynamics is found inside this loop. The symbol $\circ$ indicates the 1:2 strong resonance at which the torus and period-doubling bifurcations meet.

Fig. 6. This curve forms the boundary of a region in the phase diagram where the asymmetric periodic orbits grow in amplitude when $V_{H}$ is increased, at fixed stiffness $K$, from the "first" Hopf bifurcation point before decreasing and disappearing at the "second" Hopf bifurcation. Fig. 7(a) shows a representative bifurcation diagram for $K=0.14$, for which the asymmetric periodic orbits are computed from the Hopf bifurcation points with $V_{H}$ as the continuation parameter. In Fig. 8, two examples of direct time integration of the system (19) with (35) performed with the MatLaB routine ode23s are presented. Starting from an initial condition close to the symmetric equilibrium, they show that the trajectory is initially attracted to the asymmetric equilibrium, following the unstable eigen direction perpendicular to the flow invariant symmetric subspace. Then, oscillations grow until reaching the stable asymmetric periodic orbit. For $V_{H}$ large enough, typical stick-slip oscillations are obtained. The shear stress shows a saw-tooth shape corresponding to a slow stress build-up approximately linear in time (the interfaces slip slowly with the same order of magnitude) followed by a very fast stress drop associated with a rapid acceleration the fast interface. During this phase, the state of the fast interface becomes very small (much less than the regularisation constant $c$ ), compared to the slow interface's state which stays of order 0.1 .

When the stiffness is further reduced, for $K<0.2$, the Hopf bifurcation from the symmetric equilibrium occurs. These symmetric periodic orbits, although they are stable 
a

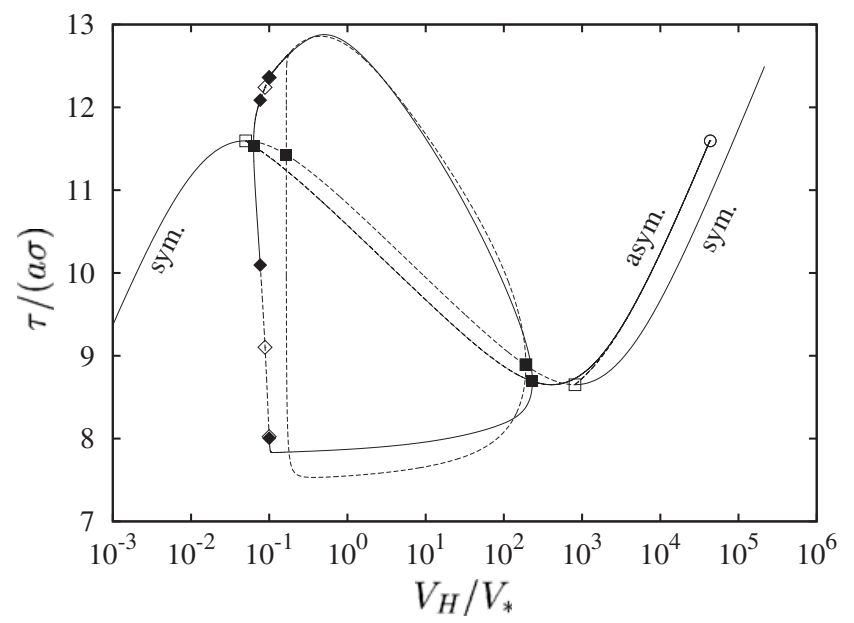

b

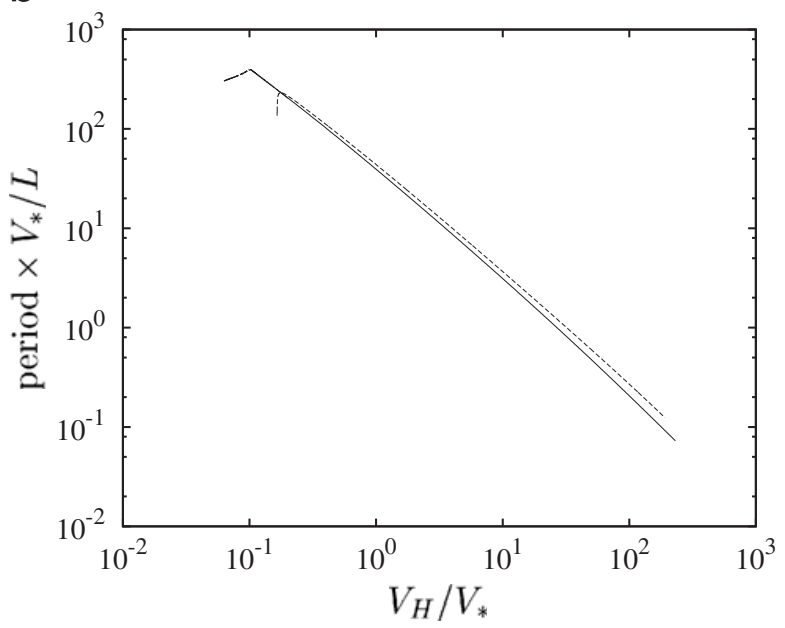

Fig. 7. (a) Bifurcation diagram for $K=0.14$ : maximum and minimum amplitudes of shear stress on the periodic orbits; saddle-node bifurcation $\circ$, pitchfork bifurcation $\square$, Hopf bifurcation $\boldsymbol{\square}$, torus bifurcation $\bullet$, period doubling bifurcation $\diamond$; solid lines are stable, dashed ones unstable. (b) Periods of the oscillatory solutions; asymmetric solution, - - - - symmetric solution.

within the subspace $\psi_{1}=\psi_{2}$, are unstable to asymmetric perturbations and have been followed by continuation as for the asymmetric limit cycles. We observe that the amplitudes of the asymmetric periodic orbits are slightly smaller than the symmetric ones, and that these two types of oscillatory state evolve in a similar fashion. Fig. 7(b) shows that the periods of the orbits are very similar as well. This reflects again the fact that the twointerface system behaves like the single-interface one as soon as $V_{H}$ is large enough, say larger than the critical $V_{H}$ at which the symmetric periodic orbit appears.

The symmetric limit cycles would be physically of little interest if they were always unstable. But, when the stiffness is decreased still further $(K<0.135)$, we find that the symmetric periodic orbits stably coexist with the asymmetric periodic orbits. The numerics are difficult to perform with AUTO at these small values of $K$. Because the symmetric and asymmetric periodic orbits come very close to each other and are then difficult to distinguish graphically, a sketch of the generic bifurcation diagram at small $K$ is presented in Fig. 9. Interestingly, the Hopf bifurcation points (labelled $\mathrm{H}_{\mathrm{a}}^{1,2}$ in Fig. 9) on the asymmetric equilibrium are not joined by a monotonically varying branch, as before. The asymmetric periodic orbits created at $\mathrm{H}_{\mathrm{a}}^{1,2}$ disappear to become symmetric orbits at pitchfork bifurcations, labelled $\mathrm{PF}^{1,2}$ respectively. In particular, note that the maximum stress on the asymmetric periodic orbit starting from $\mathrm{H}_{\mathrm{a}}^{2}$ lies very close to that on the symmetric orbit and connects to it in a subcritical manner after having become unstable at the saddle-node bifurcation SN. On the contrary, the behaviour of the asymmetric limit cycle born from $\mathrm{H}_{\mathrm{a}}^{1}$ is more straightforward and is a consequence of the narrow neighbourhood of $V_{\mathrm{M}}$ where all the corresponding bifurcations can interact. As $K \rightarrow 0$, it seems that the curve of period doubling bifurcations of the asymmetric orbits (PD) is aligned very close to the pitchfork bifurcation PF. On the phase diagram (cf. Fig. 6), the dashed four-dot line depicts the locus of the pitchfork bifurcations PF which borders the domain of existence of stable symmetric periodic orbits. As the direct two-parameter continuation failed, calculations at fixed $K$ were carried out in order to locate in the $\left(V_{H}, K\right)$ plane these pitchfork bifurcations and produce the dashed four-dot line. 
a

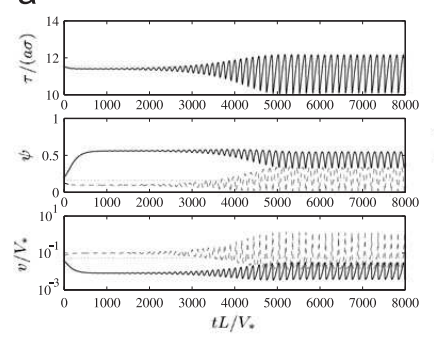

$\mathrm{b}$

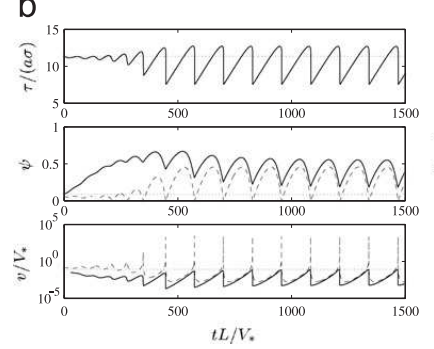

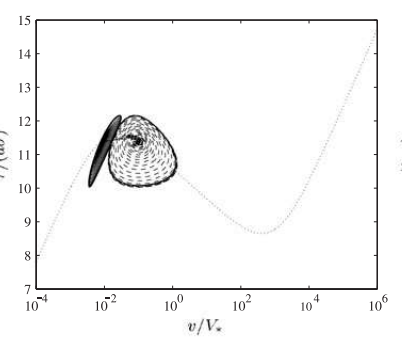

$K=0.25, V_{H} / V_{*}=0.105$

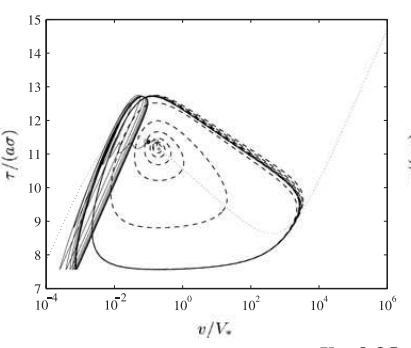

$K=0.25, V_{H} / V_{*}=0.2$

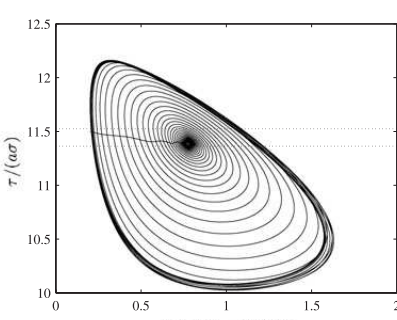

$\log \left(\psi_{1}\right)-\log \left(\psi_{2}\right)$

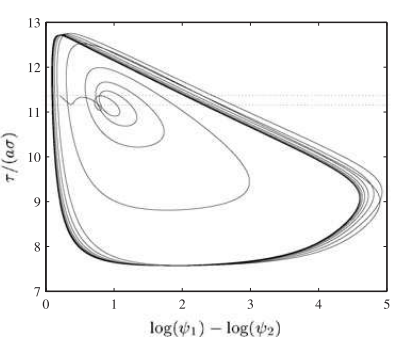

Fig. 8. Numerical time integration of the system (19) for the spinodal friction law (35). From the left to the right-hand side are plotted: the temporal evolution of the shear stress, the interfacial state and the slip rate of the two interfaces; the corresponding trajectories of the slow (dashed line) and fast (solid line) interfaces in the $(v, \tau)$-plane (the dotted line refers to the friction law); the trajectory in the asymmetric subspace $\left(\log \left(\psi_{1}\right)-\log \left(\psi_{2}\right)\right.$, $\left.\tau\right)$; the Poincaré return map of the intersections of the trajectory with the Poincaré section defined by $\tau=\tau_{\mathrm{ss}}\left(V_{H} / 2\right)$. 


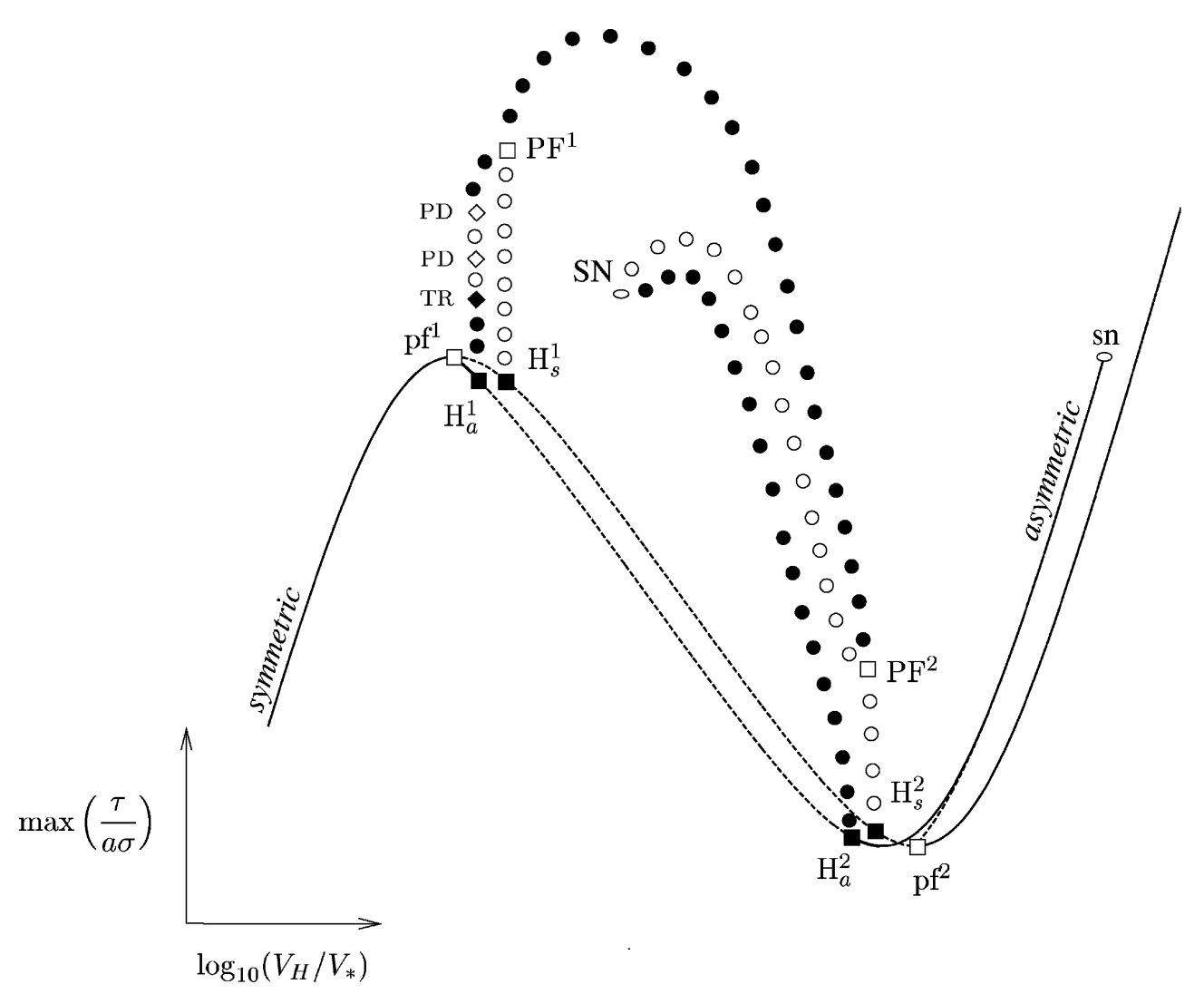

Fig. 9. Sketch of the structure of the bifurcation diagram for small $K$ : pitchfork bifurcation of equilibria (pf), Hopf bifurcations from the asymmetric equilibrium $\left(\mathrm{H}_{\mathrm{a}}^{1,2}\right)$, Hopf bifurcations from the symmetric equilibrium $\left(\mathrm{H}_{\mathrm{s}}^{1,2}\right)$, pitchfork bifurcation of periodic orbits $(\mathrm{PF})$, period doubling bifurcation of periodic orbits (PD), saddlenode bifurcation of equilibria (sn), saddle-node bifurcation of periodic orbits (SN). The circles denote the maximum of the periodic orbits: the symbol • (resp., o) represents a stable (resp., unstable) solution. The nomenclature of the bifurcation points is given at Fig. 7(a).

As suggested by the bifurcation diagram (cf. Fig. 9), the proximity of the two types of periodic orbits makes them difficult to distinguish. Fig. 10 compares the stable symmetric and asymmetric periodic orbits computed by direct time integrations for $K=0.05$ and $V_{H} / V_{*}=0.2$. Only the initial conditions are changed, the symmetric orbit being reached if the symmetry of the initial conditions is preserved. It is found indeed that these periodic orbits look very similar. Physically, the symmetric periodic orbits will be most easily observable when $K$ and $V_{H}$ are such that the asymmetric periodic orbit is unstable. Numerical time integration for $V_{H} / V_{*}=0.2$ and $K=0.01$ shows that asymmetric initial conditions lead to a limit cycle which lies in the symmetric subspace (cf. Fig. 11). As we expect from the form of Eq. (19), the periodic orbits at very small $K$ correspond to relaxation oscillations during which the "slow phase" of the dynamics lies close to the steady-state friction law $\tau_{\mathrm{ss}}(V)$.

For $K<0.216$, it is furthermore found that the asymmetric periodic orbits lose stability at a torus bifurcation (Neimark-Sacker) before becoming stable again from a torus or period-doubling bifurcation depending on the value of $K$ (see Fig. 7(a)). Numerical time integrations indicate the existence of a region of more complicated dynamics inside this loop of unstable asymmetric periodic orbits which lies between the two Hopf bifurcation curves (cf. Fig. 6 and 12(a)). Further details are provided in the next section. 

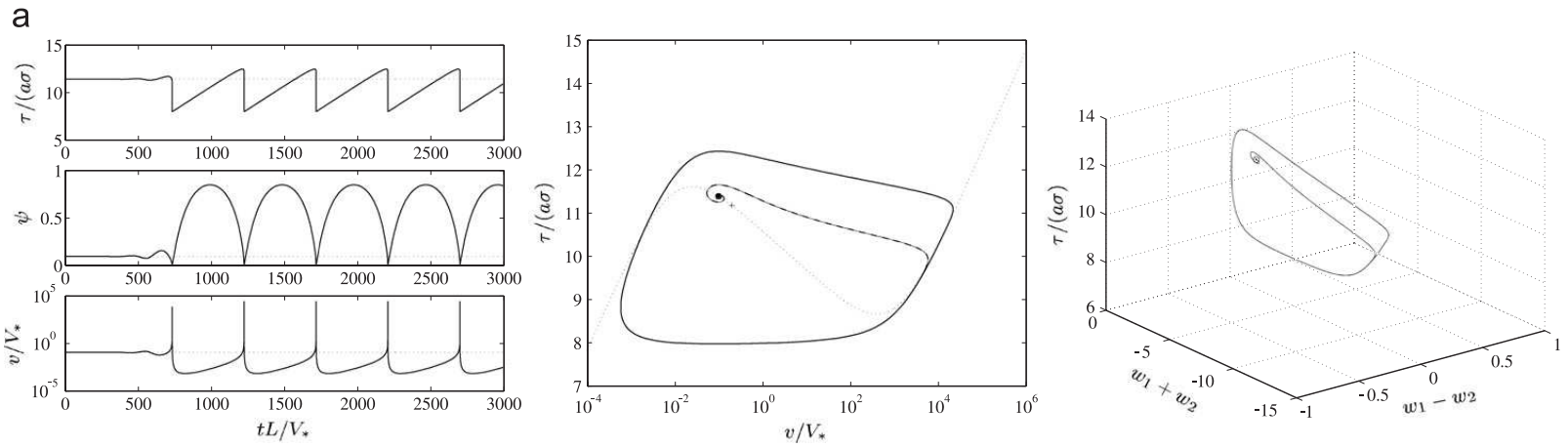

b
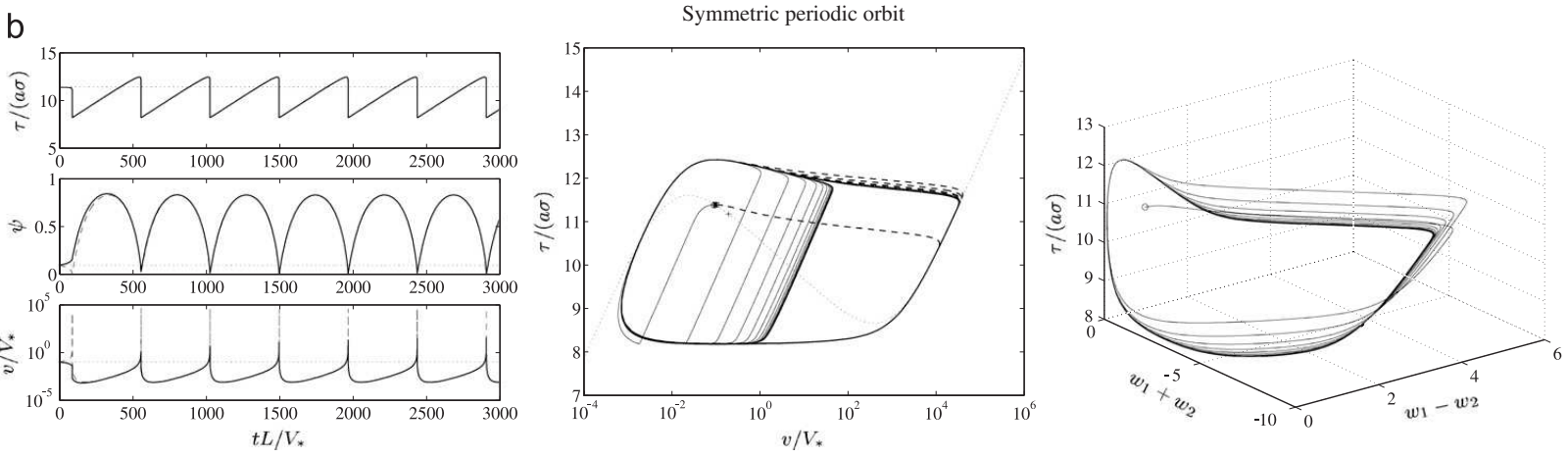

Asymmetric periodic orbit

Fig. 10. Numerical time integration of the system (19) for the spinodal friction law (35). The parameter values are $K=0.05$ and $V_{H} / V_{*}=0.2$. From the left to the right-hand side are plotted: the temporal evolution of the shear stress, the interfacial state and the slip rate of the two interfaces; the corresponding evolution of the trajectory in the variables $(v, \tau)$; and the phase space $\left(\tau, w_{1}+w_{2}, w_{1}-w_{2}\right)$. In (b) the solid and dashed lines, respectively, denote the slow and fast interfaces. The central graph clearly illustrates the differences in the maximum slip rates of the slow and fast interfaces. Both interfaces slide in a very nearly symmetric way during the slow part of the cycle. (a) Symmetric periodic orbit, (b) asymmetric periodic orbit. 

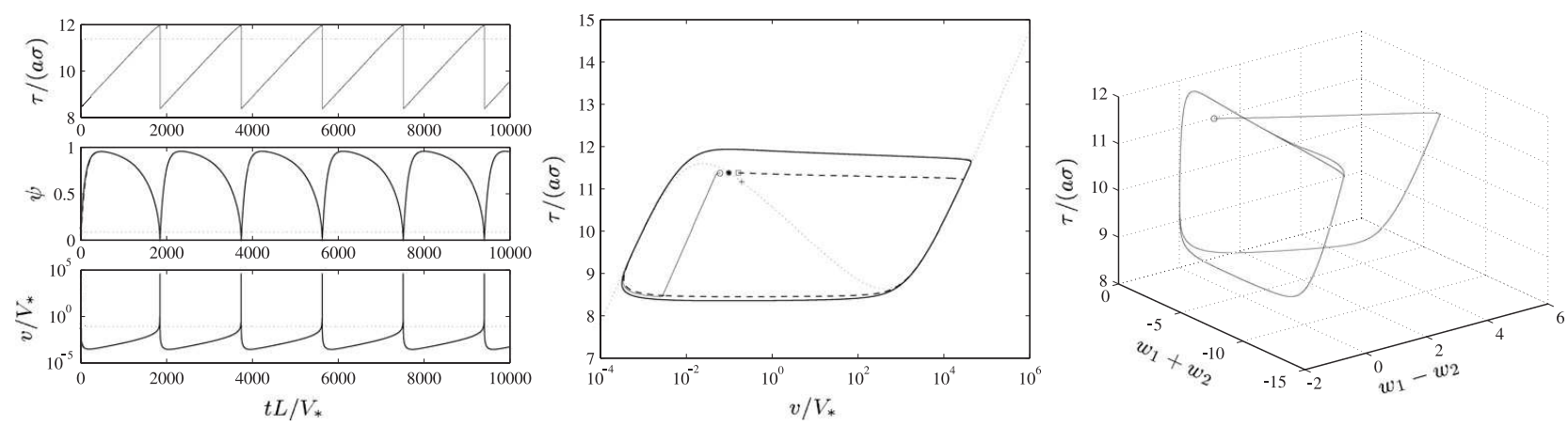

Fig 11. Numerical time integration of the two-interface system (19) for the spinodal friction law (35). The parameter values are $K=0.01$ and $V_{H} / V_{*}=0.2$. From the left to the right-hand side are plotted: the temporal evolution of the shear stress, the interfacial state and the slip rate of the two interfaces; the corresponding evolution of the trajectory in the variables $(v, \tau)$ (solid and dashed lines, respectively, denote the slow and fast interfaces); and the phase space $\left(\tau, w_{1}+w_{2}, w_{1}-w_{2}\right)$. The trajectories converge to a symmetric periodic orbit from an asymmetric initial condition. 


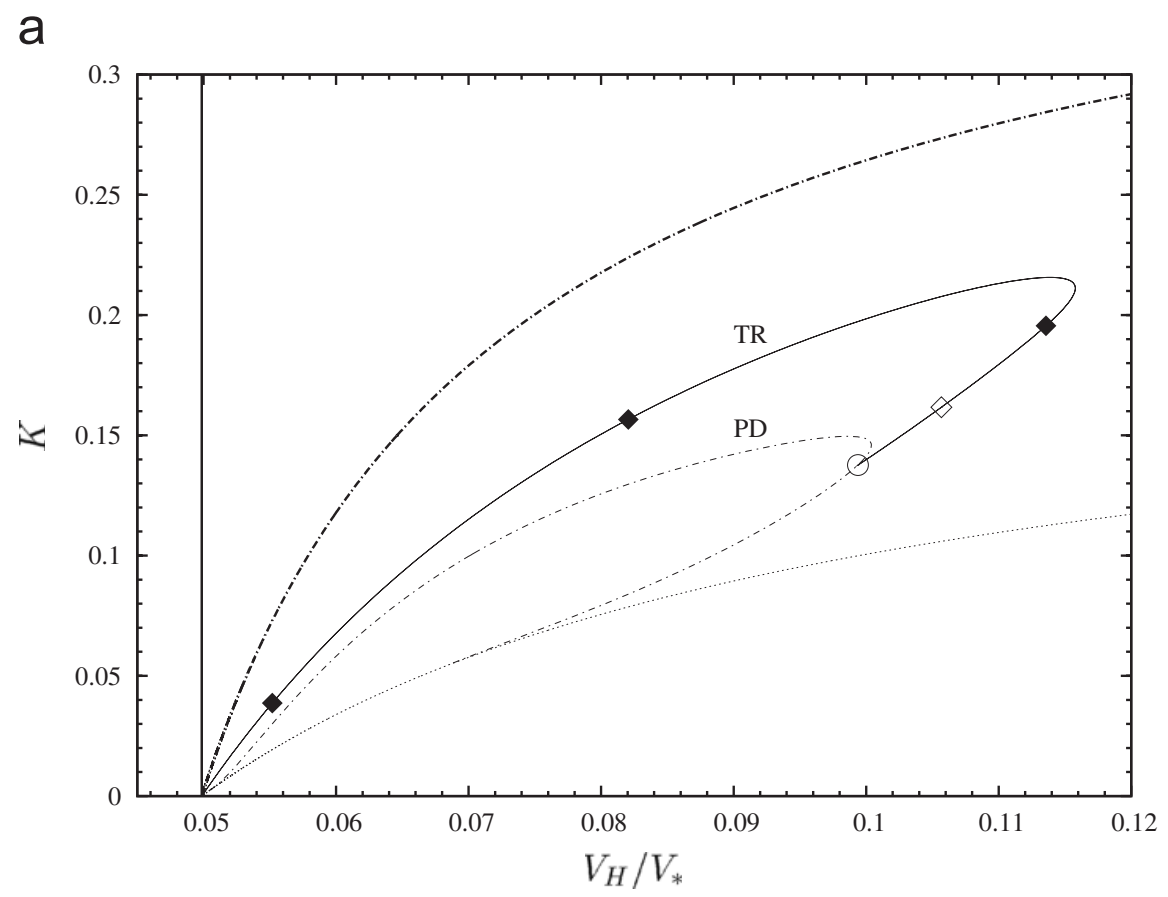

b

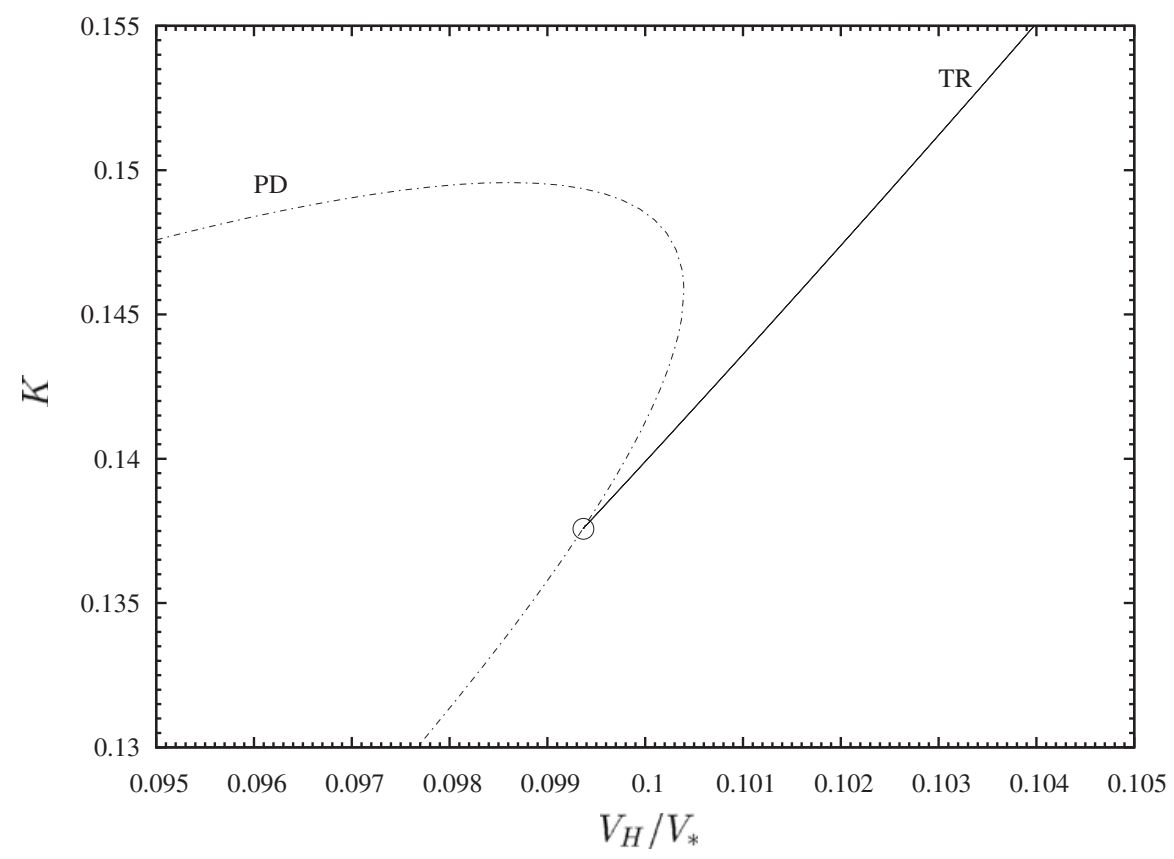

Fig. 12. Bifurcation curves in the $\left(V_{H}, K\right)$ plane. (a) Detail for small $V_{H}$ and $K$ : torus bifurcation from the asymmetric periodic orbit (TR); period-doubling bifurcation from the asymmetric periodic orbit (PD). The symbols $\diamond, \diamond, \circ$, respectively, locate the 1:4, 1:3 and 1:2 strong resonances along the torus bifurcation line. (b) Enlargement of (a) near the codimension-two point where 1:2 strong resonance occurs. The same nomenclature as Fig. 6 is used.

\subsection{The torus bifurcation and strong resonances}

\subsubsection{The torus bifurcation}

For fixed $K<0.216$, numerical continuation of the periodic orbits generated at the asymmetric Hopf bifurcation reveals that they lose stability as $V_{H}$ is increased (see Figs. 7(a) and 12(a)). The stability of the periodic orbit is determined by its Floquet multipliers, 
analogous to the eigenvalues of an equilibrium point. One Floquet multiplier is always +1 corresponding to neutral stability to perturbations in the direction tangent to the periodic orbit. The other two Floquet multipliers are either real or form a complex conjugate pair. The instability of the periodic orbit occurs along the curve labelled TR in Fig. 12(a) where a complex conjugate pair of Floquet multipliers crosses the unit circle in a Neimark-Sacker bifurcation.

The usual approach (see for example the textbooks by Guckenheimer and Holmes, 1986; Glendinning, 1994; or Wiggins, 2003) to the analysis of this torus bifurcation is to consider the "return map" given by the repeated intersection of trajectories with a Poincare section (two-dimensional plane) $\Sigma$ that intersects the periodic orbit transversely at a point. The original periodic orbit then corresponds to a fixed point for the return map. The Poincare section chosen in this work corresponds to the horizontal plane $\tau=\tau_{\mathrm{ss}}\left(V_{H} / 2\right)$. During the numerical time integration, the intersections between the Poincare section and the trajectory are stored. The discrete sequence $\left[\tau(n), v_{1}(n), v_{2}(n)\right]$ thus obtained is then used to plot the Poincaré return map in the plane $\left[\left[v_{1}+v_{2}\right](n),\left[v_{1}+v_{2}\right](n+1)\right]$. Fig. 13 gives an example of such return maps for $K=0.2$ just after the torus bifurcation has occurred. Successive intersections with the Poincare section clearly show how the trajectory winds around the torus; as a consequence, the extrema of the stress and slip rate are modulated in time.

By analogy with the Hopf bifurcation in continuous time, one might expect the appearance of an invariant circle in the plane, around which trajectories rotate with constant frequency, corresponding to winding around a torus in the original threedimensional phase space (cf. Fig. 13). The details of the Neimark-Sacker bifurcation are more subtle due to frequency locking. We will summarise the dynamics very briefly here: a clear and readable account of the essential aspects is presented in many standard references, for example Sections 4.6, 4.7 and 9.5 of Kuznetsov (2004). Let the Floquet multipliers at the torus bifurcation point be $\mathrm{e}^{ \pm \imath \theta}$. The cases $\theta=\pi, 2 \pi / 3, \pi / 2$ are known as strong resonances, of order 1:2, 1:3 and 1:4, respectively (Table 1). Away from the strong resonances it can indeed be proved that a unique invariant circle exists, either subcritically or supercritically as expected. The dynamics on the invariant circle, near the bifurcation point but away from the strong resonances, are described qualitatively by the "standard map" introduced by Arnold (1965):

$$
\phi_{n+1}=\phi_{n}+\omega+\frac{\lambda}{2 \pi} \sin \left(2 \pi \phi_{n}\right)
$$

where $\phi_{n}$ is a rescaled angle coordinate along the invariant circle, and $\lambda$ is the bifurcation parameter (the Neimark-Sacker bifurcation occurs at $\lambda=0$ ). For $0 \leqslant \lambda<1$ the $(\omega, \lambda)$ plane contains a dense collection of disjoint regions of frequency locking; one for every rational $p / q$. These regions are known as "Arnold tongues."

The characteristic width of the "Arnold tongues" is $O\left(\lambda^{q}\right)$ for small $\lambda$ and on the boundary of each tongue there is a saddle-node bifurcation of periodic orbits. For $\lambda>1$ the map (37) ceases to be invertible and the dynamics becomes much more complicated: the tongues overlap (and so there are many stable periodic orbits coexisting for the same parameter values), and chaotic dynamics occurs.

It is possible in principle to determine the map (37) by performing a series of coordinate transformations, starting from the system (19) and (35); for example this would give relations between the parameters $(\lambda, \omega)$ and the original bifurcation parameters $\left(V_{H}, K\right)$. 
Author's personal copy

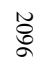
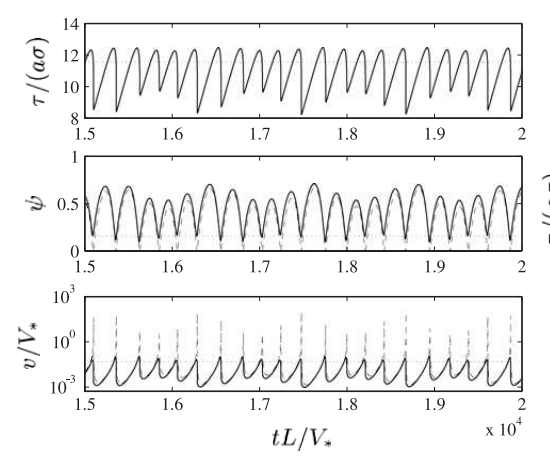
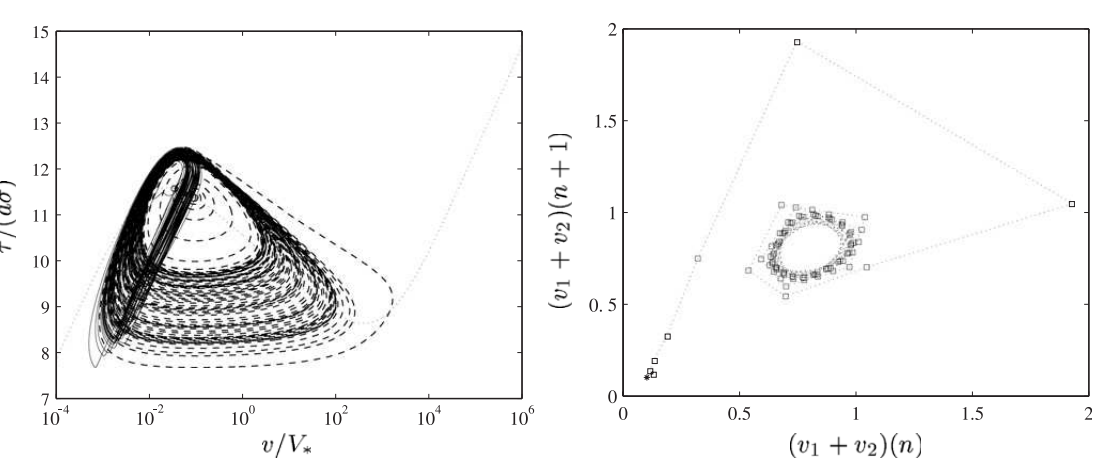

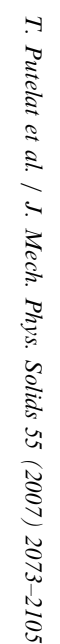


Table 1

Coordinates of the strong resonances along the torus bifurcation curve

\begin{tabular}{lrrrrr}
\hline & \multicolumn{1}{c}{$1: 4$} & \multicolumn{1}{c}{$1: 4$} & \multicolumn{1}{c}{$1: 4$} & \multicolumn{1}{c}{$1: 3$} & \multicolumn{1}{c}{$1: 2$} \\
\hline $\log \left(V_{H} / V_{*}\right)$ & -1.2582 & -1.0859 & -0.9447 & -0.9761 & -1.0027 \\
$V_{H} / V_{*}$ & 0.0552 & 0.0821 & 0.1136 & 0.1057 & 0.0994 \\
$K$ & 0.0387 & 0.1566 & 0.1956 & 0.1617 & 0.1376 \\
\hline
\end{tabular}

But this detailed information is of little interest for the purpose of these pages since the general scenario of frequency locking is well-known.

\subsubsection{The 1:2 strong resonance}

Fig. 14 shows that the angle $\theta$ of the Floquet multipliers does not remain constant along the torus bifurcation curve TR in Fig. 12. In particular it passes through points of strong resonance, where the solid curve in Fig. 14 crosses the two horizontal thin dotted lines labelled 1:4 and 1:3. The detailed analysis of these strong resonances is more complicated; the dynamics are not described by the one-dimensional map (37). A careful analysis of the 1:4 and 1:3 strong resonances shows that the bifurcation curve TR exists on both sides of the bifurcation point, and the gradient of the curve in Fig. 12 remains finite at these points. However, this is not the case as the Floquet multipliers approach the point of 1:2 resonance at $K \approx 0.138$, where $\theta / \pi \rightarrow 1$.

The detailed analysis of the 1:2 resonance case is also due to Arnold (1983) (see also Kuznetsov, 2004, Section 9.5.3). We very briefly outline the method of analysis here. At the 1:2 resonance point the linearisation of the return map at the fixed point corresponding to the original periodic orbit has two Floquet multipliers of -1 . In Jordan standard form the linearisation takes the form

$$
\left(\begin{array}{l}
x_{n+1} \\
y_{n+1}
\end{array}\right)=\left(\begin{array}{cc}
-1 & 1 \\
0 & -1
\end{array}\right)\left(\begin{array}{l}
x_{n} \\
y_{n}
\end{array}\right)+\text { nonlinear terms }
$$

using coordinates $(x, y)$ on the Poincaré section $\Sigma$, since in the generic case there will only be a single eigenvector for the eigenvalue -1 . The second iterate of this map is therefore

$$
\left(\begin{array}{l}
x_{n+2} \\
y_{n+2}
\end{array}\right)=\left(\begin{array}{cc}
1 & -2 \\
0 & 1
\end{array}\right)\left(\begin{array}{l}
x_{n} \\
y_{n}
\end{array}\right)+\text { nonlinear terms. }
$$

In both cases the nonlinear terms can be simplified by the process of putting the map into normal form; successive near-identity coordinate changes are made that remove nonlinear terms in the Taylor series order by order. Using Picard iteration it turns out to be possible (see, for example Kuznetsov, 2004, Section 9.5) to construct a continuous-time dynamical system for which the unit-time evolution along trajectories is conjugate to the dynamics of the map (38). Such a pair of differential equations is referred to in the literature as an "approximating system." In the case of 1:2 resonance the approximating system is the normal form for an equilibrium point with a double-zero eigenvalue, often referred to as the Takens-Bogdanov bifurcation (Takens, 1974; Holmes and Rand, 1980). In addition, the approximating system naturally inherits a symmetry admitting only odd terms in this case. After rescalings, the approximating system takes the form (including terms only up to 


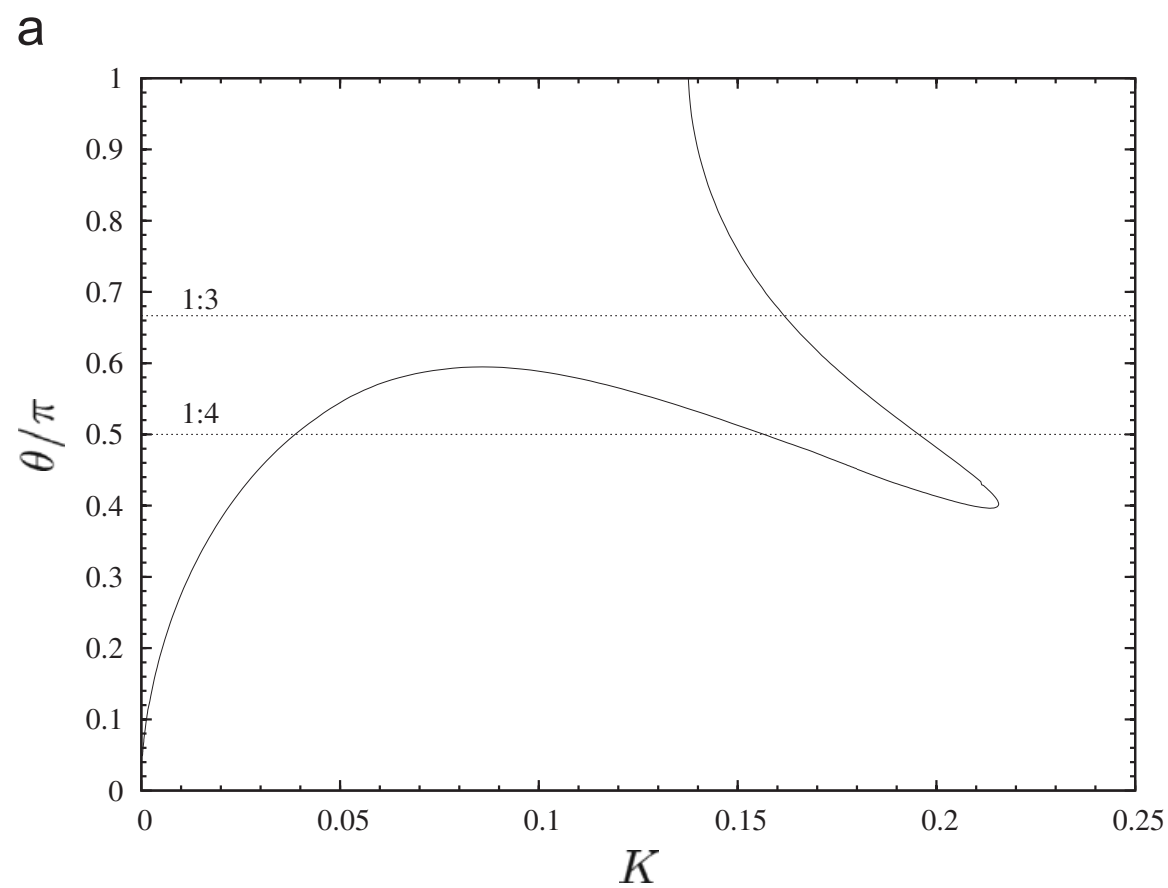

b

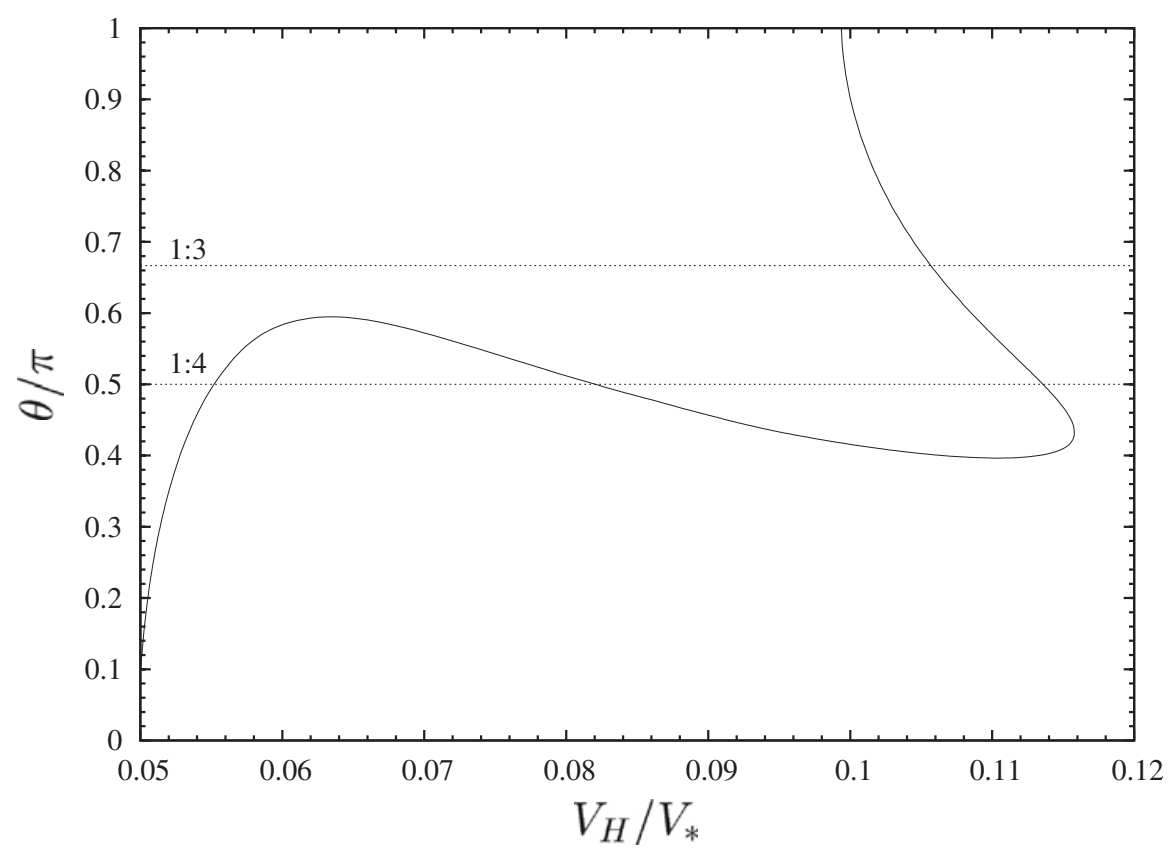

Fig. 14. Variation of angle $\theta$ of Floquet multipliers $\mathrm{e}^{\mathrm{i} i \theta}$ along the torus bifurcation curve TR in Fig. 12(a). Horizontal dotted lines indicate points of $1: 3\left(\theta / \pi=\frac{2}{3}\right)$ and $1: 4\left(\theta / \pi=\frac{1}{2}\right)$ resonance along the curve. The point of $1: 2$ resonance corresponds to $\theta / \pi=1$ and is reached at $V_{H} / V_{*}=0.0994, K_{\mathrm{c}}=0.1376$.

cubic order)

$$
\begin{aligned}
& \dot{v}=w, \\
& \dot{w}=-\mu_{1} w-\mu_{2} v-v^{3}-v^{2} w .
\end{aligned}
$$

The bifurcation structure implied by this analysis, in our case, is presented in Fig. 15. In reality the bifurcation structure found in the approximating system will not be reflected precisely in the original ODEs due to the influence of higher-order terms that are not in 
a

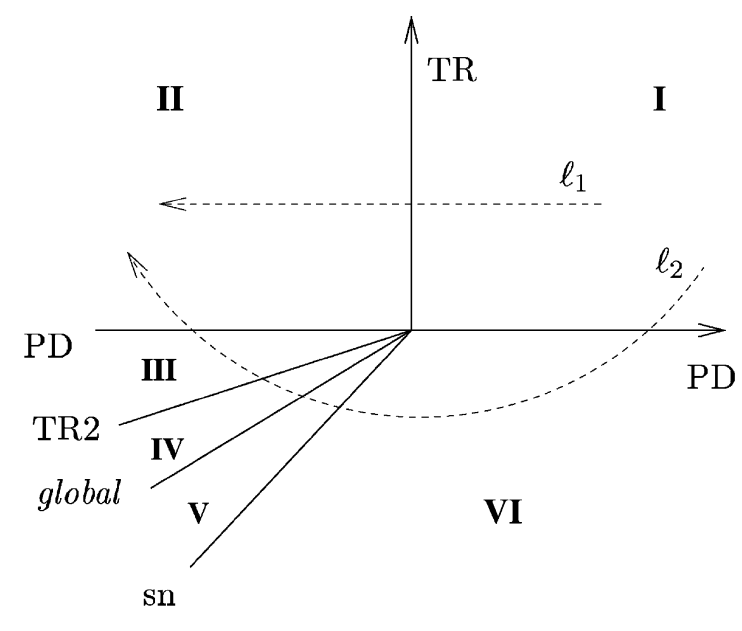

b
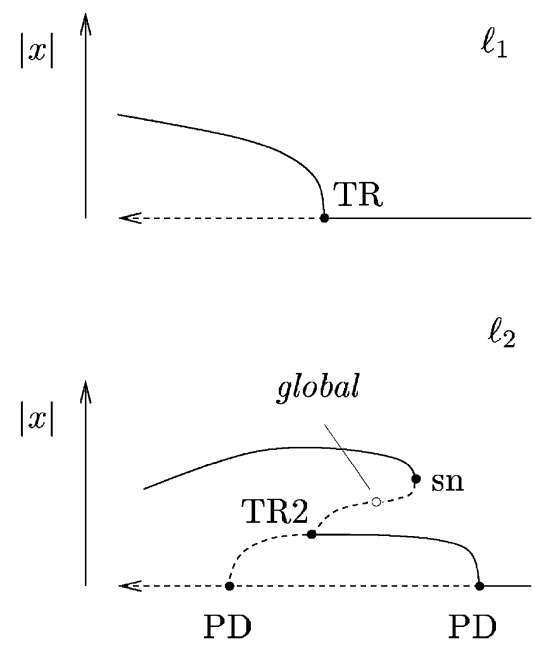

Fig. 15. (a) Bifurcation curves in the $\left(\mu_{1}, \mu_{2}\right)$ plane for the approximating system (39)-(40), interpreted in terms of the dynamics near the 1:2 resonance point. There are six regions of qualitatively distinct dynamics, separated by the curves labelled: (TR) torus bifurcation, (PD) period-doubling, (TR2) torus bifurcation of the period-doubled orbit, (sn) saddle-node bifurcation of tori, (global) global bifurcation of tori. (b) Illustrative bifurcation diagrams along the paths $\ell_{1}$ and $\ell_{2}$ indicated in (a).

normal form. The existence of a global bifurcation, in particular, indicates that complicated dynamics, due to homoclinic tangles, will be present near the strong resonance point.

Numerical simulations do indeed show the existence of complicated dynamics, and a plethora of stable periodic orbits, in the region bounded by the torus and period-doubling curves in Fig. 12. For example, Fig. 16 gives the results for $K=0.14$ of the numerical time integration of the two-interface equations close to a 1:4 strong resonance. In particular, Fig. 16(a) shows the subcritical nature of the torus bifurcation. Fig. 16(b) presents a fourfold structure in the Poincare return map which is explained by the proximity of the 1:4 strong resonance point. The corresponding trajectory makes three little loops which produce small amplitude oscillations in the shear stress. Then the trajectory makes a long excursion characterised by a rapid stress drop due to the acceleration of the fast interface. Then the stress builts up slowly, the interfaces being rough again.

Continuation of solutions and location of bifurcation points is made more difficult by the proximity of the lower branch of the PD curve to the curve of Hopf bifurcations from the symmetric equilibrium. It appears that the symmetric branch of periodic orbits that is produced is not involved in the bifurcation structure, but since the asymmetric orbits often lie close to the symmetric subspace $\psi_{1}(t)=\psi_{2}(t)$ this proximity is a further source of numerical difficulties.

\section{Conclusion}

We have carried out an extensive study of stability of the steady- state and oscillatory slidings of two parallel frictional interfaces, which divide horizontally an elastic body of finite thickness, and which is sheared at a constant velocity. Linear stability analysis, numerical continuation and time-integration techniques have been used to examine the nonlinear dynamical system (19) which describes the two-interface sliding dynamics. 
a

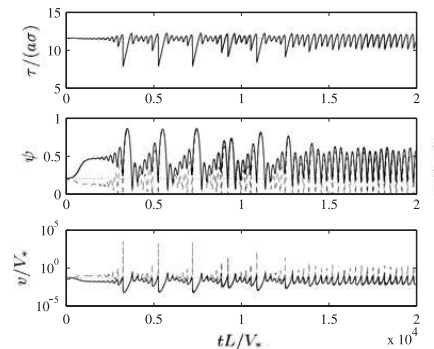

b

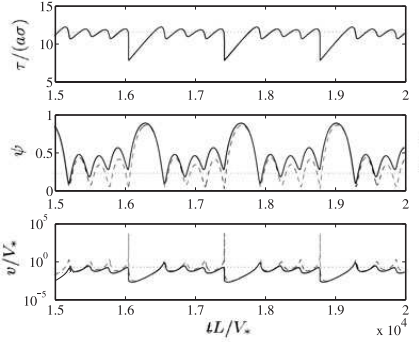

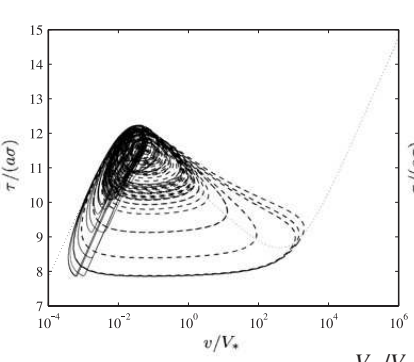

$V_{H} / V_{*}=0.075$

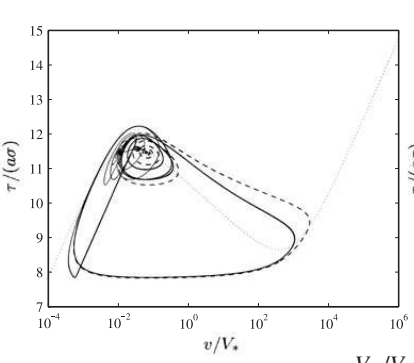

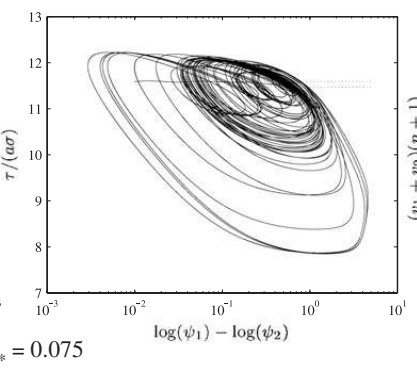

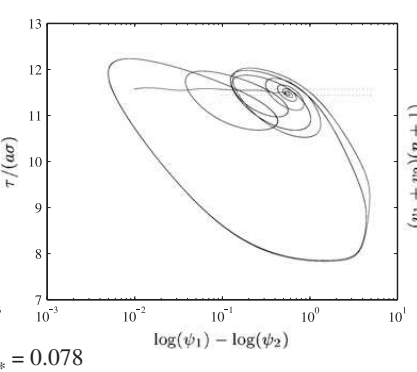

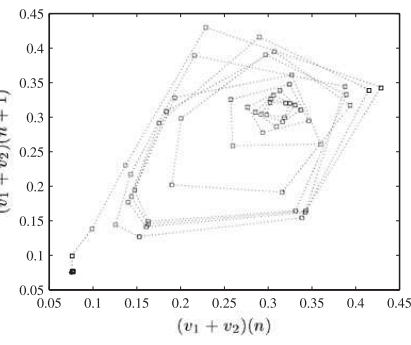

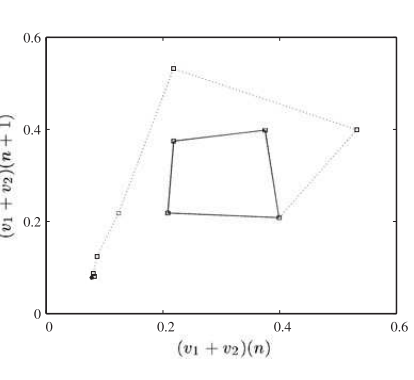

Fig. 16. Numerical time integration of the two-interface system (19) for the spinodal friction law (35). The value of the dimensionless stiffness is $K=0.14$. From the left to the right hand side are plotted the temporal evolution of the shear stress, the interfacial state and the slip rate of the two interfaces; the corresponding trajectories of the slow and fast interfaces in the $(v, \tau)$-plane; the Poincare return map of the intersections of the trajectory with the Poincare section defined by $\tau=\tau_{\mathrm{ss}}\left(V_{H} / 2\right)$. Critical values of $V_{H} / V_{*}$ at the torus and period-doubling bifurcation: TR $=0.0767, \mathrm{PD}=0.0842, \mathrm{PD}=0.0980, \mathrm{TR}=0.1000$. 
Restricting the analysis to the framework of rate-and-state friction, conditions for the existence of stable steady-state slidings have been obtained. Assuming only horizontal displacement and neglecting wave propagation, thus making the shear stress homogeneous, we showed that the (non-)monotonicity of the steady-state friction law is an essential feature of the problem, governing the number and the nature of the possible stationary slidings of the two interfaces.

If the friction coefficient is a monotonic function of the interfacial slip rate, only one symmetric steady state, in the sense that the interfaces slip with the same velocity, is allowed. Independently of the system stiffness and thus unlike the single interface situation, this symmetric sliding is unstable if the friction law is velocity weakening. As a result, the weaker interface is selected and the sliding is localised along it, the more resistive interface creeping slower and slower.

In the case of a non-monotonic friction law, an asymmetric steady state with one slow and one fast interface can exist in addition to the symmetric solution. The existence and stability of these solutions are determined by two parameters: the driving velocity $V_{H}$ and the stiffness $k$ of the system defined as the ratio of the shear modulus $G$ and the total thickness $H$ of the medium. Analytical results for stability show how the symmetric steady state bifurcates via pitchfork bifurcation to the asymmetric steady state at critical values of the driving velocity equal to twice the slip rates at which the friction coefficient reaches an extremum. The shape of the friction law determines the super- or subcritical nature of this pitchfork bifurcation. Furthermore, if the stiffness is small enough, both the symmetric and the asymmetric equilibria undergo Hopf bifurcations. The resulting periodic orbits coexist stably over a substantial region of parameter space. Complex dynamics originate from the interaction between the pitchfork and Hopf bifurcations of the symmetric equilibria. The interaction generates a secondary oscillatory instability (a torus bifurcation), frequency locking, and strong resonances. The existence domains of these various possible states of the two-interface system are shown on the phase diagram in Fig. 6. Figs. 7(a) and 9, respectively, represent typical bifurcation diagrams for moderate and small values of the dimensionless stiffness $K=L k /(a \sigma)$.

Due to its three-dimensional phase space, the two-interface problem is much richer than the single interface one. The range of possible behaviours is governed by the qualitative form of the friction law: any law with the same "spinodal" structure as the law we proposed should admit similar dynamics. That all of the complex dynamics occurred at low values of $V_{H} / V_{*}$ and $K$ in the present work is a consequence of the parameter values that were chosen. The continuation package Auto proved to be a particularly useful tool for the computation of slip rates, interfacial states, and overall to obtain the "macroscopic" friction law $\tau\left(V_{H}\right)$.

Considering moreover that the friction law (35) synthesises the physics and concepts of rate-and-state friction and greatly improves the Dieterich-Ruina type friction law, we hope that our two-interface system would motivate experimental investigations whose results could be confronted with the theoretical predictions we report in this paper. Such experiments will, for instance, provide estimates for the two additional parameters $\left(t_{* *}\right.$ and $\left.c\right)$ from which the non-monotonicity of the friction law originates.

As a result, such experimental results, coupled to our theoretical insights, should offer a better understanding of the transitions between the velocity weakening and strengthening behaviours of friction. At the micromechanical level, the very low speed transition between behaviours would illustrate the competition between static and dynamic asperity 
interactions, whereas the high speed transition between behaviours might enlighten the (nonlinear) role of the normal pressure and its relation to the parameter $c$. It has indeed been observed (Kilgore et al., 1993) that a change in normal pressure is associated with the occurrence of a weakening/strengthening transition in dry rock friction.

\section{Acknowledgements}

This work has been supported from the Postdoctoral Marie-Curie Individual Fellowship (Contract No. HPMF-CT 2002-01587) and the European Research Network DE.F.I.NO, Deformation and Fracture Instabilities in Novel Materials and Processes (RTN/DEFINO, Dec. 2002-Nov. 2006, HPRN-CT-2002-00198). JHPD is supported by Newnham College, Cambridge.

We wish to thank Coralia Cartis, Alain Cochard, Herbert Huppert, Arieh Iserles, Jean Schmittbuhl, Toshihiko Shimamoto, and Michael Thompson for useful discussions and comments.

\section{Appendix A. Location of the roots of the cubic $P_{3}(s)$}

Let us consider the cubic equation (30) written as

$$
-\left(a_{3}+s / k\right)\left(s-s_{1}\right)\left(s-s_{2}\right)=\left(a_{1} s_{1}+a_{2} s_{2}\right) s-s_{1} s_{2}\left(a_{1}+a_{2}\right),
$$

where $a_{1}, a_{2}$ and $s_{3}$ are, respectively, defined by (31)-(33). The cubic of the left hand side of the previous equation is denoted $Q_{3}(s)$ and the straight line expression on the right-hand side $L(s)$. Considering a solution $V_{1} \neq V_{2}$, the problem is to find a criterion on $k$ determining when $P_{3}$ becomes unstable (i.e. when $P_{3}$ has a zero with a positive real part at least). This is equivalent to looking for the intersection points between $Q_{3}(s)$ and $L(s)$.

Let us here assume that $s_{1}>0$ and $s_{2}<0$ as considered in Section 3.2.2. This implies that the cubic $Q_{3}$ resembles the sketch in Fig. 17 because $Q_{3}(0)=-a_{3} s_{1} s_{2}>0$, and $a_{1}>0$ and $a_{2}<0$. We then know that $L(s)$ is an increasing function of $s$ because $a_{1} s_{1}+a_{2} s_{2}>0$. Furthermore, we showed in Section 3.2.1 that the solution $V_{1} \neq V_{2}$ only exists if the steady-state friction law has a velocity strengthening branch steeper than the weakening one. In other words, it requires that $-\tau_{V}^{(1)}<\tau_{V}^{(2)}$ with the convention $s_{1}>0$. This implies that

$$
-1 / \tau_{V}^{(1)}-1 / \tau_{V}^{(2)}=a_{1}+a_{2}-a_{3}>0,
$$

and then that

$$
a_{1}+a_{2}>0 \text {, }
$$

because $a_{3}>0$. As a consequence, we always have

$$
L(0)=-s_{1} s_{2}\left(a_{1}+a_{2}\right)>Q_{3}(0)>0 .
$$

In addition, it is straightforward to show that $s_{2}<s_{0}<0$ where $s_{0}$ is the intersection of $L(s)$ with the abscissa. As we consider a given solution $V_{1} \neq V_{2}$ obtained for a given choice $V_{H}$, $s_{1}, s_{2}$ and the $a_{i}$ are fixed and so is the line $L(s)$. Fig. 17 then shows the geometrical configuration of the problem. Thus, by only changing the value of $k$, we move the location of the third root $-k a_{3}$ of $Q_{3}(s)$, modifying the number of possible intersections of $Q_{3}(s)$ and $L(s)$. Let us suppose we increase $k$ from 0. From Fig. 17, it is clear that there firstly exist two unstable roots of $P_{3}$ which become complex conjugated, but still with a positive 


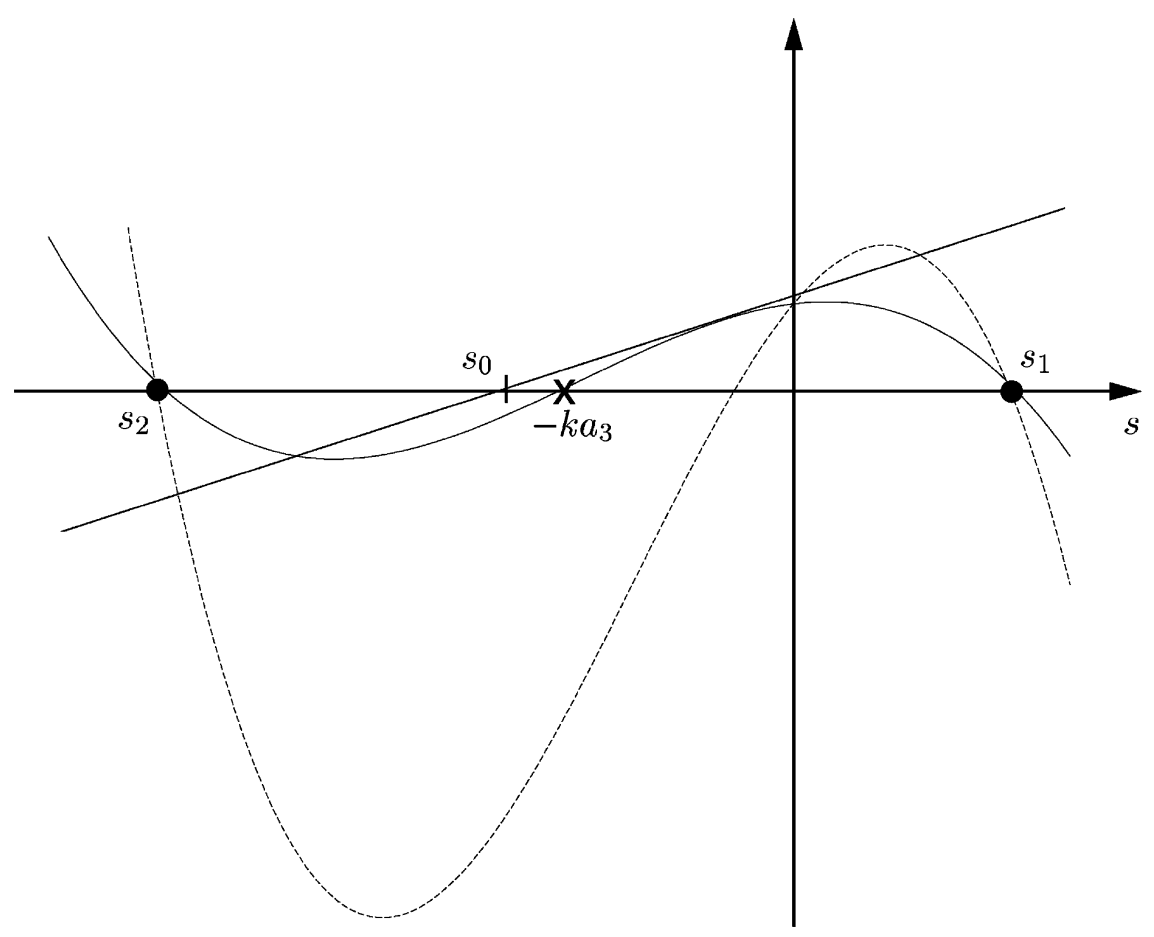

Fig. 17. Location of the roots of $P_{3}$ : the solid straight line - denotes $L(s)$, the solid curve - denotes $Q_{3}(s)$ for some $k$ so that $Q_{3}(s)$ and $L(s)$ are tangent. The dashed curve - - - illustrates the cubic $Q_{3}(s)$ obtained for a smaller $k$. Increasing $k$ from 0 geometrically shows that the most unstable roots of the cubic equation (30) are complex conjugates.

real part, when $k$ reaches a value at which $Q_{3}(s)$ is tangential to $L(s)$. Increasing $k$ continuously then shows that these complex conjugate roots cross the imaginary axis and become stable until they are real again at the next point of tangency. As a consequence, we geometrically know that there exists a critical stiffness $k_{\mathrm{c}}^{*}$ at which the most unstable roots of the polynomial $P_{3}$ become pure imaginary. Note furthermore that $Q_{3}(0)<L(0)$ prevents a real root to become positive by varying $k$. Let us then find $k_{\mathrm{c}}^{*}$ in order that $\mathrm{i} \omega$ is a root of $P_{3}$. The real and imaginary parts of $P_{3}(\mathrm{i} \omega)=0$ lead to the set of equations

$$
\begin{aligned}
& -\left(s_{1}+s_{2}-k a_{3}\right) \omega^{2}=-s_{1} s_{2}\left(a_{1}+a_{2}-a_{3}\right) k, \\
& \omega^{2}=k[\underbrace{s_{1}\left(a_{1}-a_{3}\right)+s_{2}\left(a_{2}-a_{3}\right)}_{\text {denoted } c}]+s_{1} s_{2} .
\end{aligned}
$$

Note that $c>0$. The first equation implies that $s_{1}+s_{2}-k a_{3}<0$ so that $\omega$ exists and is real. The stiffness then must be greater than another critical value

$$
k_{\mathrm{c}}^{* *}=\left(s_{1}+s_{2}\right) / a_{3} .
$$

Under this condition, the elimination of $\omega^{2}$ from the two equations gives the quadratic equation

$$
c a_{3} k^{2}+\left[s_{1} s_{2}\left(a_{1}+a_{2}\right)-c\left(s_{1}+s_{2}\right)\right] k-s_{1} s_{2}\left(s_{1}+s_{2}\right)=0,
$$

that the critical stiffness $k_{\mathrm{c}}^{*}$ must satisfy. The discriminant of (42), a quadratic form in $s_{1}^{2}$ and $s_{2}^{2}$, is

$$
\Delta=\left(a_{1}-a_{3}\right)^{2} s_{1}^{4}+2\left[-a_{3}^{2}+\left(a_{1}+a_{2}\right) a_{3}+a_{1} a_{2}\right] s_{1}^{2} s_{2}^{2}+\left(a_{2}-a_{3}\right)^{2} s_{2}^{4},
$$


and must be positive to allow the solutions to be real

$$
k_{ \pm}=\frac{1}{2}\left[\frac{s_{1}+s_{2}}{a_{3}}-\frac{s_{1} s_{2}\left(a_{1}+a_{2}\right)}{c a_{3}}\right] \pm \frac{1}{2} \frac{\sqrt{\Delta}}{c a_{3}} .
$$

Note that if $s_{1}+s_{2}<0$, it would be easy to show that (42) has only one positive solution $k_{+} \equiv k_{\mathrm{c}}^{*}$. The criterion of stability of $P_{3}$ then is

$$
k>k_{\mathrm{c}}^{*} \text {. }
$$

\section{References}

Arnold, V.I., 1965. Small denominators I. Mappings of the circumference onto itself. Trans. AMS 42, $213-284$. Arnold, V.I., 1983. Geometrical Methods in the Theory of Ordinary Differential Equations. Springer, Berlin.

Ben-Zion, Y., Rice, J.R., 1997. Dynamic simulations of slip on a smooth fault in an elastic solid. J. Geophys. Res. $102,17,771-17,784$.

Bowden, F.P., Tabor, D., 1954. The Friction and Lubrication of Solids. Clarendon Press, Oxford.

Carlson, J.M., Batista, A.A., 1996. Constitutive relation for the friction between lubricated surfaces. Phys. Rev. E $53,4153-4165$.

Dieterich, J.H., 1978. Time-dependent friction and the mechanics of stick-slip. Pure Appl. Geophys. 116, 790-806.

Dieterich, J.H., 1979. Modeling rock friction: 1. Experimental results and constitutive equations. J. Geophys. Res. 84, 2161-2168.

Doedel, E.J., Keller, H.B., Kernevez, J.P., 1991. Numerical analysis and control of bifurcation problems. Int. J. Bifur. Chaos 1(3), 493-520. AUTO 2000 available via Internet from 〈http://indy.cs.concordia.ca/auto/〉.

Estrin, Y., Bréchet, Y., 1996. On a model of frictional sliding. Pure Appl. Geophys. 147, 745-762.

Glendinning, P., 1994. Stability, Instability, and Chaos. Cambridge University Press, Cambridge.

Gu, J.C., Rice, J.R., Ruina, A.L., Tse, S.T., 1984. Slip motion and stability of a single degree of freedom elastic system with rate and state dependent friction. J. Mech. Phys. Solids 32, 167-196.

Guckenheimer, J., Holmes, P., 1986. Nonlinear Oscillations, Dynamical Systems and Bifurcations of Vector Fields. Springer, Berlin.

Heslot, F., Baumberger, T., Perrin, B., Caroli, B., Caroli, C., 1994. Creep, stick-slip, and dry-friction dynamics: Experiments and a heuristic model. Phys. Rev. E 49, 4973-4988.

Holmes, P.J., Rand, D.A., 1980. Phase portraits and bifurcations of the nonlinear oscillator $\ddot{x}+\left(\alpha+\gamma x^{2}\right) \dot{x}+\beta x+\delta x^{3}=0$. Int. J. Nonlinear Mech. 15, 449-458.

Iooss, G., Joseph, D.D., 1980. Elementary Stability and Bifurcation Theory. Undergraduate Texts in Mathematics. Springer, Berlin.

Kilgore, B.D., Blanpied, M.L., Dieterich, J.H., 1993. Velocity dependent friction of granite over a wide range of conditions. Geophys. Res. Lett. 20 (10), 903-906.

Kuznetsov, Y.A., 2004. Elements of Applied Bifurcation Theory. Applied Mathematical Sciences Series, third ed. Springer, Berlin.

Lapusta, N., Rice, J.R., Ben-Zion, Y., Zheng, G., 2000. Elastodynamic analysis for slow tectonic loading with spontaneous rupture episodes on faults with rate-and-sate dependent friction. J. Geophys. Res. 105 (B10), 23,765-23,789.

Lim, Y.F., Chen, K., 1998. Dynamics of dry friction: A numerical investigation. Phys. Rev. E 58, 5637-5642.

Marone, C., 1998. Laboratory-derived friction laws and their application to seismic faulting. Annu. Rev. Earth Sci. 26, 643-696.

Nakatani, M., 2001. Conceptual and physical clarification of rate and state friction: frictional sliding as a thermally activated rheology. J. Geophys. Res. 106 (B7), 13,347-13,380.

Putelat, T., Willis, J.R., 2004. Frictional sliding of a multislip system. In: Gutkowski, W., Kowalewski, T.A. (Eds.), Mechanics of the 21st Century, Proceedings of the 21st International Congress of Theoretical and Applied Mechanics, 15-21 August, Warsaw, Poland. Springer, Berlin. Available on CDROM or at $\langle\mathrm{http}: / /$ ictam04.ippt.gov.pl//.

Rabinowicz, E., 1995. Friction and Wear of Materials, second ed. Wiley-Interscience, New York. 
Rice, J.R., 2006. Heating and weakening of faults during earthquake slip. J. Geophys. Res. 111, B05311, doi:10.1029/2005JB004006.

Rice, J.R., Ben-Zion, Y., 1996. Slip complexity in earthquake fault models. Proc. Natl. Acad. Sci. USA 93, 3811-3818.

Rice, J.R., Ruina, A.L., 1983. Stability of steady frictional slipping. J. Appl. Mech. 50, 343-349.

Rice, J.R., Lapusta, N., Ranjith, K., 2001. Rate and state dependent friction and the stability of sliding between elastically deformable solids. J. Mech. Phys. Solids 49, 1865-1898.

Ruina, A.L., 1980. Friction laws and instabilities: a quasistatic analysis of some dry frictional behavior. Ph.D. thesis, Brown University.

Ruina, A.L., 1983. Slip instability and state variable friction laws. J. Geophys. Res. 88, 10,359-10,370.

Scholz, C.H., 1998. Earthquakes and friction law. Nature 391, 37-42.

Scholz, C.H., 2002. The Mechanics of Earthquakes and Faulting, second ed. Cambridge University Press, Cambridge.

Shimamoto, T., 1986. Transition between frictional slip and ductile flow for halite shear zones at room temperature. Science 231, 711-714.

Takens, F., 1974. Forced oscillations and bifurcations. Comm. Math. Inst., Rijksuinversiteit Utrecht 3, 1-59.

Tsutsumi, A., Shimamoto, T., 1997. High-velocity frictional properties of gabbro. Geophys. Res. Lett. 24 (6), 699-702.

Weeks, J.D., 1993. Constitutive laws for high-velocity frictional sliding and their influence on stress drop during unstable slip. J. Geophys. Res. 98, 17,637-17,648.

Weeks, J.D., Tullis, T.E., 1985. Frictional sliding of dolomite: a variation in constitutive behavior. J. Geophys. Res. 90, 7821-7826.

Wiggins, S., 2003. Introduction to Applied Nonlinear Dynamical Systems and Chaos. Texts in Applied Mathematics, second ed. Springer, Berlin. 